\title{
ER stress-mediated autophagy promotes Myc-dependent transformation and tumor growth
}

Lori S. Hart, ${ }^{1}$ John T. Cunningham, ${ }^{2}$ Tatini Datta, ${ }^{1}$ Souvik Dey, ${ }^{1}$ Feven Tameire, ${ }^{1}$ Stacey L. Lehman, ${ }^{1}$ Bo Qiu, ${ }^{1}$ Haiyan Zhang, ${ }^{1}$ George Cerniglia, ${ }^{1}$ Meixia Bi, ${ }^{1}$ Yan Li, ${ }^{3}$ Yan Gao, ${ }^{3}$ Huayi Liu, ${ }^{1}$ Changhong Li, ${ }^{4}$ Amit Maity, ${ }^{1}$ Andrei Thomas-Tikhonenko, ${ }^{5}$ Alexander E. Perl, ${ }^{6}$ Albert Koong, ${ }^{7}$ Serge Y. Fuchs, ${ }^{8}$ J. Alan Diehl, ${ }^{3}$ lan G. Mills, ${ }^{9,10,11}$ Davide Ruggero, ${ }^{2}$ and Constantinos Koumenis ${ }^{1}$

${ }^{1}$ Department of Radiation Oncology, University of Pennsylvania School of Medicine, Philadelphia, Pennsylvania, USA. ${ }^{2}$ School of Medicine and Department of Urology, Helen Diller Family Comprehensive Cancer Center, UCSF, San Francisco, California, USA. ${ }^{3}$ Department of Cancer Biology and Abramson Family Cancer Research Institute, University of Pennsylvania School of Medicine,

Philadelphia, Pennsylvania, USA. “Department of Pediatrics and 5Department of Pathology and Laboratory Medicine, The Children's Hospital of Philadelphia, Philadelphia, Pennsylvania, USA. ${ }^{6}$ Division of Hematology/Oncology, University of Pennsylvania, Philadelphia, Pennsylvania, USA.

${ }^{7}$ Department of Radiation Oncology, Stanford University School of Medicine, Stanford, California, USA. ${ }^{8}$ Department of Animal Biology,

University of Pennsylvania School of Veterinary Medicine, Philadelphia, Pennsylvania, USA. ${ }^{9}$ Prostate Cancer Research Group, Centre for Molecular Medicine Norway, University of Oslo, Oslo, Norway. ${ }^{10}$ Department of Urology, and ${ }^{11}$ Department of Cancer Prevention, Oslo University Hospitals, Oslo, Norway.

The proto-oncogene c-Myc paradoxically activates both proliferation and apoptosis. In the pathogenic state, c-Myc-induced apoptosis is bypassed via a critical, yet poorly understood escape mechanism that promotes cellular transformation and tumorigenesis. The accumulation of unfolded proteins in the ER initiates a cellular stress program termed the unfolded protein response (UPR) to support cell survival. Analysis of spontaneous mouse and human lymphomas demonstrated significantly higher levels of UPR activation compared with normal tissues. Using multiple genetic models, we demonstrated that c-Myc and N-Myc activated the PERK/ eIF2 $\alpha /$ ATF 4 arm of the UPR, leading to increased cell survival via the induction of cytoprotective autophagy. Inhibition of PERK significantly reduced Myc-induced autophagy, colony formation, and tumor formation. Moreover, pharmacologic or genetic inhibition of autophagy resulted in increased Myc-dependent apoptosis. Mechanistically, we demonstrated an important link between Myc-dependent increases in protein synthesis and UPR activation. Specifically, by employing a mouse minute $\left(\mathrm{L24}^{+/-}\right)$mutant, which resulted in wild-type levels of protein synthesis and attenuation of Myc-induced lymphomagenesis, we showed that Myc-induced UPR activation was reversed. Our findings establish a role for UPR as an enhancer of c-Myc-induced transformation and suggest that UPR inhibition may be particularly effective against malignancies characterized by c-Myc overexpression.

\section{Introduction}

The unfolded protein response (UPR) is a cellular homeostatic program initiated by an excess of unfolded/misfolded client proteins in the ER lumen, with a primarily cytoprotective effect. There are 3 ER-resident proximal sensors of unfolded proteins and ER stress: PKR-like ER stress kinase (PERK), activating transcription factor 6 (ATF6), and inositol-requiring transmembrane kinase and endonuclease 1 (IRE1) (1-3). Under steady-state conditions, these stress sensors are bound by the chaperone protein GRP78 (BiP). The accumulation of unfolded proteins results in the dissociation of GRP78 and the subsequent activation of PERK, ATF6, and IRE1 $(4,5)$. Active PERK phosphorylates eukaryotic initiation factor 2-alpha (eIF2 $\alpha$ ) at Ser51, preventing the exchange of GDP for GTP, effectively blocking the initiation of protein synthesis during cellular recovery from ER stress (6). While general protein synthesis is limited, the translation of specific mRNAs is induced, including that of the transcription factor ATF4 (7). Stresses in the tumor microenvironment (e.g., low oxygen, decreased amino acid availability, and low glucose) activate components of the UPR, the abrogation of which leads to inhibition of tumor progression (8-13). In addi-

Conflict of interest: The authors have declared that no conflict of interest exists. Citation for this article: J Clin Invest. 2012;122(12):4621-4634. doi:10.1172/JCI62973. tion to microenvironmental stressors, the loss of tumor suppressor function, specifically of the tuberous sclerosis complex genes (TSC1, TSC2), has also been associated with UPR activation (14). Collectively, these findings reveal a link between UPR activation and adaptation to tumor microenvironmental stress.

Another cellular adaptation mechanism induced by both normal and transformed cells is autophagy, a self-digestive cellular stress response that enables cells to catabolize and recycle proteins, cytosolic components, and organelles to promote survival (15). Common cellular stresses known to induce autophagy include amino acid starvation, glucose withdrawal, and ER stress. The regulation of autophagy is accomplished primarily by the autophagyrelated (Atg) proteins, organized into multiple complexes and acting at various stages within the pathway. Activation of a specific major complex (the ULK1 [Atg1] kinase complex) leads to a signaling cascade that culminates in the formation of the autophagosome, a double-membrane vesicle that encloses the cellular contents destined for degradation. Once fully formed, the autophagosomes fuse with lysosomes, resulting in the degradation and recycling of their contents. Although autophagy may play a complex role in tumorigenesis and tumor resistance to therapy (16-18), Atg proteins have been proposed as attractive targets for cancer therapy $(18,19)$. Cells under ER stress have been shown to 
activate macro-autophagy, and it has been suggested that at least part of the autophagosomal membrane originates from enlarged ER membrane $(20,21)$ and is dependent on an active PERK/eIF2 $\alpha$ pathway (22). Recently, it was shown that in response to hypoxic stress, PERK-dependent increases in ATF4 lead to prolonged autophagic flux, contributing to hypoxic adaptation (10).

Cell-autonomous insults, including oncogene activation, are known to activate cellular stress responses, including metabolic stress, apoptosis, DNA damage responses, and cellular senescence. Therefore, transformed cells often acquire a concurrent activation of pro-survival pathways (23-25). In particular, the MYC oncogene, which is the target of chromosomal translocation and gene amplification during the development of many human cancers, is known to induce both increased proliferation and apoptosis, depending on the cellular context (26-28). c-Myc functions as a transcription factor and induces proliferation through the upregulation of genes required for cell cycle progression (cyclin $\mathrm{D}$, cyclin E), energy metabolism (lactate dehydrogenase A, GLUT1) (29), and ribosome biogenesis (RNA polymerase III, ribosomal proteins) (30). The latter has been associated with an increased rate of protein synthesis, which is required for MYC oncogenic activity and survival (31-33). However, the particular pathways under Myc-dependent translational control and the underlying mechanism by which this occurs remain elusive.

Based on the ability of the Myc oncogene to induce considerable increases in both ribosome biogenesis and rates of protein synthesis (reviewed extensively in refs. 34, 35), we hypothesized that oncogenic activation of c-Myc during transformation would inflict ER stress and elicit UPR activation. Moreover, we reasoned that such an induction of the UPR would be cytoprotective and thus promote Myc-dependent tumorigenesis. Using several inducible Myc cell models as well as genetic and pharmacologic tools, we show that Myc induction leads to activation of the PERK/eIF2 $\alpha /$ ATF4 axis of the UPR, resulting in increased autophagy and protection against ER-dependent apoptosis. Moreover, we demonstrate that robust upregulation of the UPR occurs in human lymphoma samples and mouse lymphoma models, suggestive of a role for UPR activation in the pathogenesis of disease. Therefore, we have identified a cell-autonomous role for PERK as a molecular switch regulating autophagy and apoptosis in response to Myc activation, and have uncovered PERK as a therapeutic target for the treatment of Myc-associated malignancies.

\section{Results}

c-Myc activation initiates PERK-dependent UPR signaling that is attenuated by chemical chaperones. We hypothesized that c-Myc activation increases the protein load of the ER and activates cytoprotective UPR signaling. To test this, we employed two cell lines with regulated c-Myc expression: the human P493-6 B cell line, in which c-Myc expression is repressed in the presence of tetracycline (Tet-off $\mathrm{c}-\mathrm{Myc}$ ), and immortalized mouse embryonic fibroblasts (MEFs) stably expressing the tamoxifen-inducible c-Myc chimera mycER. P493-6 cells were cultured in the presence or absence of tetracycline, and UPR activation was analyzed by immunoblot analysis for eIF $2 \alpha$ phosphorylated at serine 51 (p-eIF $2 \alpha$ ). In the absence of tetracycline, P493-6 cells expressed robust levels of c-Myc, which correlated with high levels of p-eIF $2 \alpha$ (Figure 1A) and mRNA levels of the c-Myc target ornithine decarboxylase $(O D C)$ (Supplemental Figure 1A; supplemental material available online with this article; doi:10.1172/JCI62973DS1). Addition of tetracycline resulted in rapid and nearly complete c-Myc downregulation within 4 hours, followed by a decrease in the levels of p-eIF $2 \alpha$ by 24 hours, while total eIF $2 \alpha$ levels remained unchanged. The delay between the loss of c-Myc expression and decreased p-eIF $2 \alpha$ is likely due to the time required for recovery from the presence of unfolded proteins in the ER. When cells were cultured in tetracycline-free medium, c-Myc levels rebounded rapidly, which coincided with increased levels of p-eIF2 $\alpha, O D C$ mRNA, and cell size (Figure 1A, Supplemental Figure 1, A and B, and ref. 31). When cells in which c-Myc was repressed (24-hour tetracycline treatment) were treated with the SERCA pump inhibitor and ER stress inducer thapsigargin, eIF2 $\alpha$ phosphorylation was increased, demonstrating that cells lacking c-Myc expression maintain intact UPR signaling.

To further analyze UPR induction, we measured the nuclear accumulation of the downstream UPR target ATF4 (Figure 1B). As with p-eIF2 $\alpha$, the level of nuclear ATF4 correlated with c-Myc expression, indicative of UPR activation specifically through PERK. Spliced X-box binding protein-1 (XBP1s) transcript level was also reduced by $50 \%$ when c-Myc expression was inhibited (Figure 1C). Taken together, these results suggest that both the PERK and IRE1 arms of the UPR pathway are activated in human lymphoma cells in response to c-Myc upregulation. To test whether these findings extend to other Myc family members, we used two neuroblastoma cell lines with differentially regulated N-Myc: tamoxifen-inducible N-MycER activation in human neuroblastoma SHEP cells (36) and tetracycline-inducible N-Myc expression in Tet-21 SHEP cells (37). Activation of N-Myc caused upregulation of the UPR targets ATF4 and XBP1s in N-MycER SHEP cells (Figure 1D) and eIF2 $\alpha$ phosphorylation in Tet-21 SHEP cells (Supplemental Figure 1C).

To test whether PERK is required for c-Myc-dependent UPR activation, we used SV40-immortalized MEFs from Perkflfl mice, in which the Perk gene is flanked by loxP sites, permitting Perk excision following infection with Ad-Cre, a Cre recombinase-expressing adenovirus. The MEFs were transduced with a retroviral vector expressing a mycER fusion protein to establish cells (mycER:Perk $k^{f / f l}$ ) with tamoxifen-activated c-Myc. As expected, Myc activation resulted in a significant increase in protein synthesis (Supplemental Figure 2A). Similar to the UPR activation in human P493-6 cells, p-eIF2 $\alpha$ and p-PERK levels were increased following treatment with the tamoxifen analog 4-hydroxytamoxifen (4-HT) (Figure 1E). Importantly, in the absence of PERK (mycER:Perk ${ }^{-/-}$clone 2.2, Supplemental Figure $3 \mathrm{~A}$ ), eIF $2 \alpha$ phosphorylation was substantially attenuated, indicating that Myc-induced eIF2 $\alpha$ phosphorylation is PERK dependent (Figure 1E). We also measured ATF4 activation using a reporter construct with luciferase expression under control of the 5' UTR of ATF4 (38), which contains two open reading frames with opposing activity, one positive-acting and one inhibitory. Wek and colleagues demonstrated that under conditions of ER stress and eIF $2 \alpha$ phosphorylation, delays in ribosomal scanning allow for successful translation from the positive-acting reading frame, resulting in increased ATF4 protein levels (39). ATF4 was induced in mycER:Perk ${ }^{f / f l} \mathrm{MEFs}$ following either c-Myc expression or thapsigargin treatment; in contrast, the mycER:Perk ${ }^{-/-}$MEFs were deficient in ATF4 induction (Figure 1F). Collectively, these findings indicate that c-Myc activation is sufficient to activate ER stress and the PERK/eIF2 $\alpha /$ ATF 4 arm of the UPR. Unlike in P493-6 cells, we did not observe XBP1 splicing in MEFs upon Myc activation (Supplemental Figure 3B), suggesting that the IRE1/XBP1 arm of the UPR may be activated by c-Myc in a cell-specific or host-specific manner. 
A
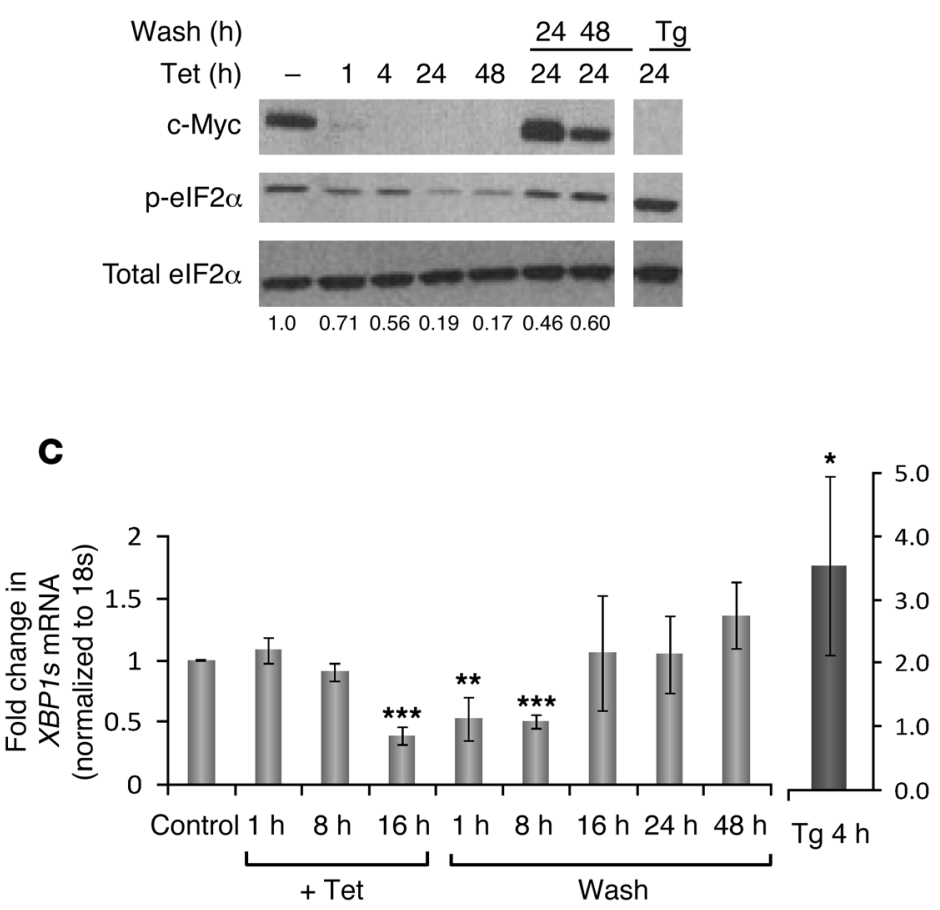

E

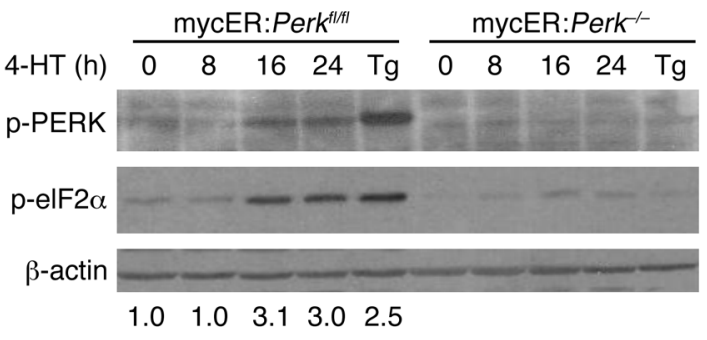

$\mathbf{G}$

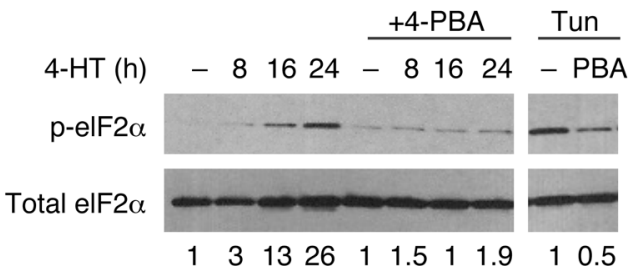

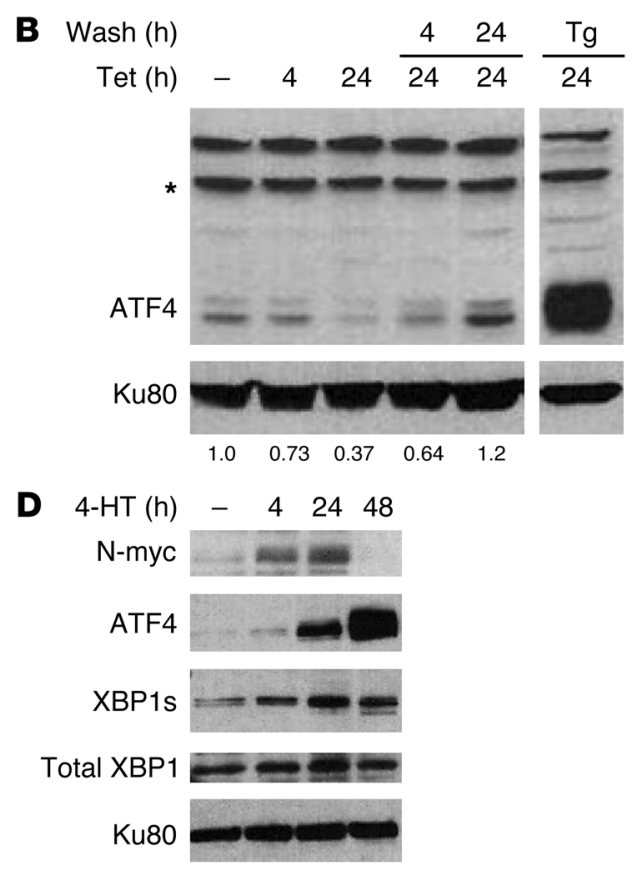

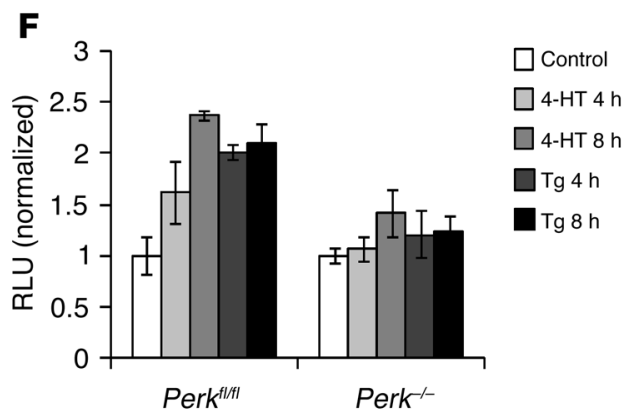

Figure 1

C-Myc-induced UPR activation in P493-6 human lymphoma cells and immortalized MEFs. (A and B) P493-6 B cells expressing Tet-repressible c-Myc were treated with tetracycline (Tet) or thapsigargin (Tg) and (A) c-Myc and phosphorylated elF2 $\alpha$ (S51) and (B) nuclear ATF4 protein levels were analyzed (immunoblots are representative of more than 3 independent experiments; *nonspecific bands on ATF4 immunoblot). Lanes were run on the same gel but were noncontiguous. Wash refers to the time $(\mathrm{h})$ following removal of tetracycline and continued culturing in tet-free media. (C) qPCR of mRNA levels of the UPR target XBP1s. Values were normalized to 18s rRNA and expressed as fold change relative to control (Tet-free) conditions ( $n=3$ independent experiments; ${ }^{* *} P<0.003$, ${ }^{* *} P<0.04,{ }^{*} P<0.05$, Student's 2 -tailed $t$ test). Scale on the right refers to Tg sample only. (D) SHEP N-MycER cells were treated with 4-HT to activate the nuclear translocation of N-Myc, and immunoblotting was performed for N-Myc, ATF4, and XBP1s. (E) MEFs were treated with 4-HT to activate c-Myc and p-PERK, and p-elF2 $\alpha$ levels were analyzed by immunoblotting. (F) MEFs expressing the 5' UTR-ATF4-luciferase construct were treated with 4-HT for the indicated times, luciferase expression was analyzed, and results were normalized to CMV-Renilla luciferase and shown as fold change over control. (G) mycER:Perk ${ }^{f / f l}$ MEFs were treated with 4-HT or tunicamycin (Tun) in the presence or absence of 4-PBA (10 mM), and p-elF2 $\alpha$ levels were analyzed (representative of 2 independent experiments). Values below blots represent total pixel intensity of p-elF2 $\alpha$ or ATF4 normalized to the loading control for each lane and are shown as fold change over each control.

To test whether c-Myc activates the UPR via the accumulation of unfolded or misfolded proteins, we co-treated mycER:Perk $f^{l / f l}$ MEFs with 4-HT and the chemical chaperone 4-phenylbutyric acid (4-PBA), which has been shown to augment protein folding and attenuate metabolic abnormalities and insulin resistance in a mouse model of diabetes (40). 4-PBA completely blocked c-Mycinduced UPR activation (Figure $1 G$ ) without significantly affecting c-Myc accumulation (Supplemental Figure 2B). As expected, 
A
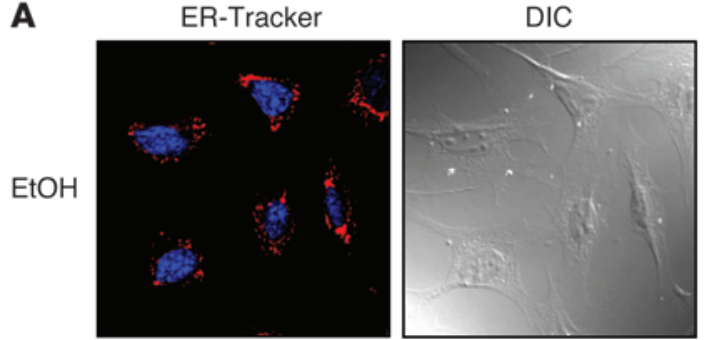

4-HT

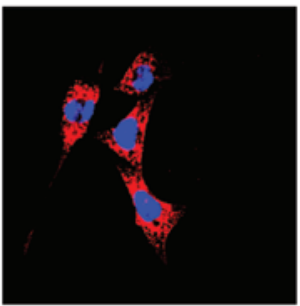

C
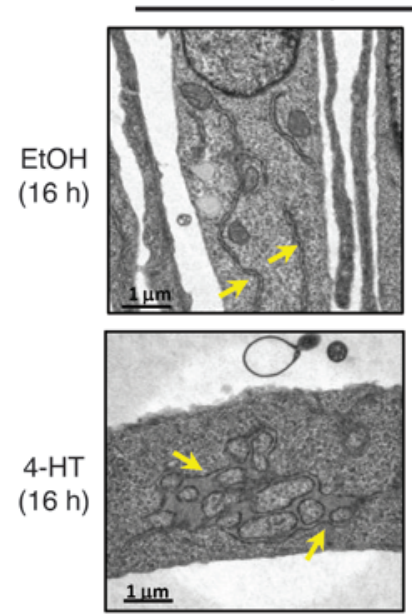

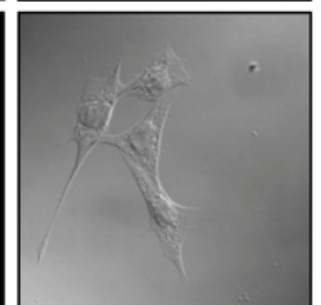

mycER:Perk ${ }^{A / H}$
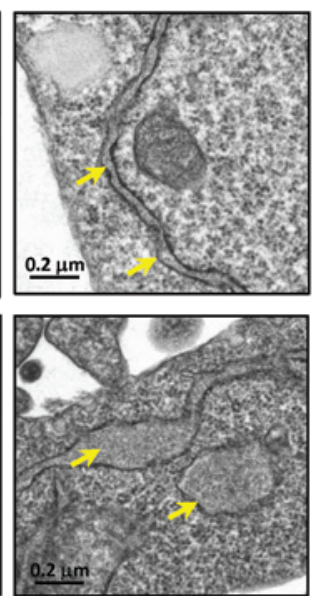

B

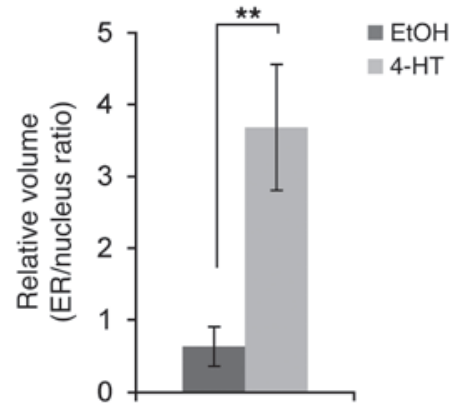

mycER:Perk ${ }^{\prime-}$
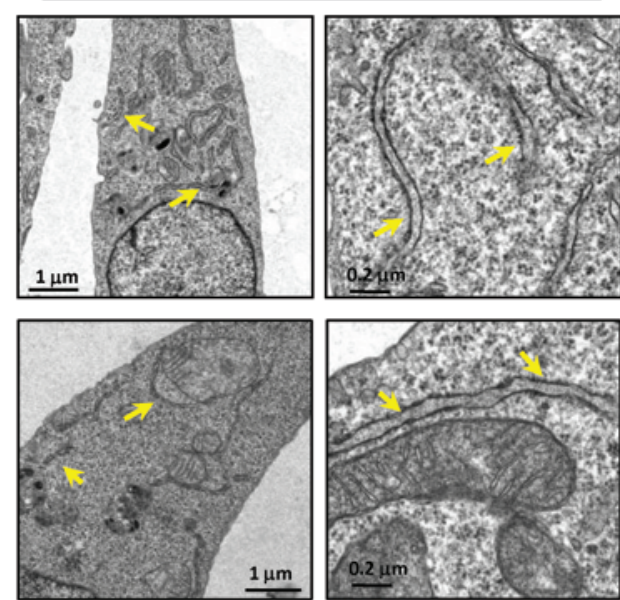

Figure 2

c-Myc expression induces PERK-dependent ER expansion. (A) mycER:Perk ${ }^{\text {flfl }}$ MEFs were treated with EtOH or 4-HT, stained with the ER-specific dye ER-Tracker, and analyzed by fluorescence confocal microscopy (cells were counterstained with DAPI; original magnification, $\times 400)$. DIC, differential interference contrast. (B) Relative ER volume (ER/nucleus) of cells stained in $\mathbf{A} .{ }^{* \star} P<0.05$, Student's 2 -tailed $t$ test. (C) mycER:Perk ${ }^{f / l t}$ and mycER:Perk ${ }^{-1}$ MEFs were treated with $4-\mathrm{HT}$, and ER structures were examined by electron microscopy. Scale bars: $1.0 \mu \mathrm{m}$ (2 left panels); $0.2 \mu \mathrm{m}$ (2 right panels) for each cell line.

4-PBA also limited the phosphorylation of eIF2 $\alpha$ induced by the glycosylation inhibitor and ER stress-inducing agent tunicamycin. These findings indicate that c-Myc-induced UPR activation is overridden by increased chaperone activity.

To further test our hypothesis that c-Myc expression activates the UPR via the induction of ER stress, we examined the morphology of the ER. Activation of c-Myc by addition of 4-HT resulted in more prominent ER morphology than in vehicle-treated control cells (Figure 2A), corresponding to a significantly higher ER/nucleus ratio (Figure $2 \mathrm{~B}$ ) and a 3 -fold increase in the fluorescence of the ER-specific probe ER-Tracker (Supplemental Figure 3C). Electron microscopy revealed a substantial increase in ER area, with clearly expanded membranes and distended lumen in mycER:Perk ${ }^{f / f l}$ cells following 16-hour treatment with 4-HT compared with control cells (Figure 2C). Moreover, flow cytometric analysis of ER-Tracker staining showed a $28 \%$ increase in ER fluorescence in human P493-6 cells with activated c-Myc (Supplemental Figure 3D).
These findings implicate c-Myc in the morphological expansion of the ER, indicative of the accumulation of misfolded proteins and dysregulated ER function in the absence of PERK.

PERK-dependent UPR signaling promotes cell survival following $c-M y c$ activation. Next, we investigated the cellular outcome of both PERKproficient and PERK-deficient MEFs when challenged with c-Myc induction. PERK ablation dramatically decreased cell survival following c-Myc activation as measured by clonogenic growth assay (Figure $3 \mathrm{~A}$ ). Immunoblot analysis revealed the upregulation of cleaved poly ADP ribose polymerase (PARP) following Myc activation, which was substantially greater in the absence of PERK (Figure 3B), indicating that induction of apoptosis was contributing to decreased clonogenicity in PERK-deficient cells. Cell cycle analysis revealed a Myc-induced increase in the fraction of cells in $S$ phase (decreased $\mathrm{G}_{1} / \mathrm{S}$ ratio) in both mycER:Perk ${ }^{l / f l}$ and mycER:Perk ${ }^{-/}$cells (Supplemental Figure 2C). Results from examination of morphological changes with phase contrast microscopy and sub- $\mathrm{G}_{1}$ content further 
A

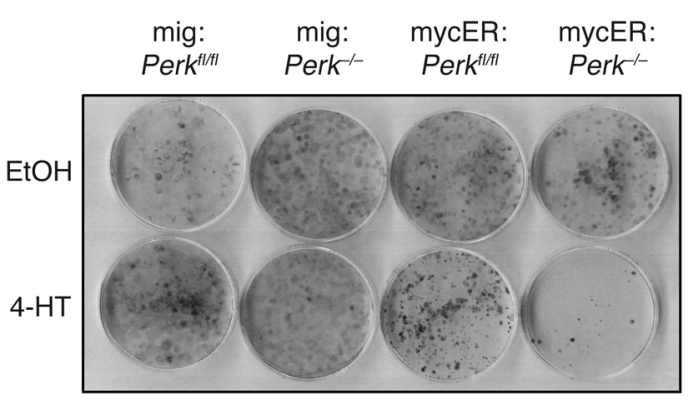

B mig: mig: mycER: mycER:

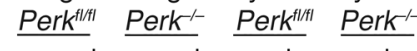
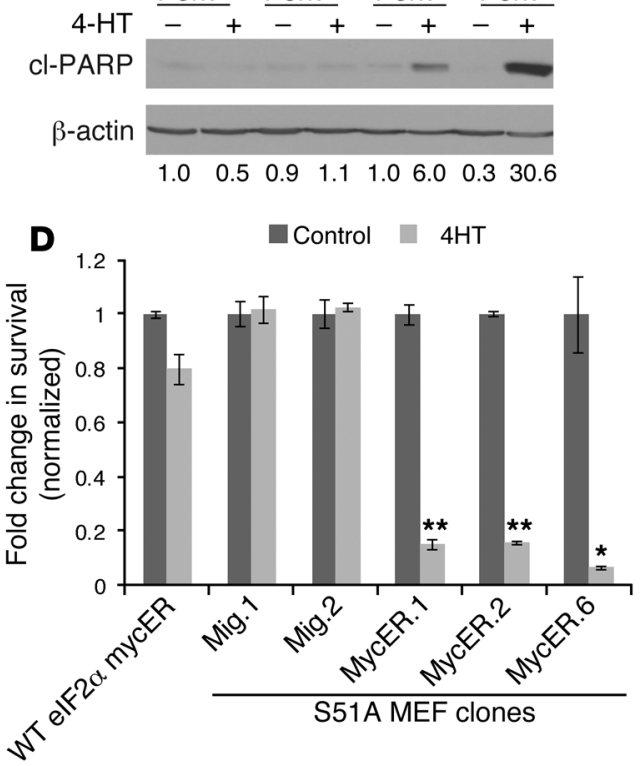

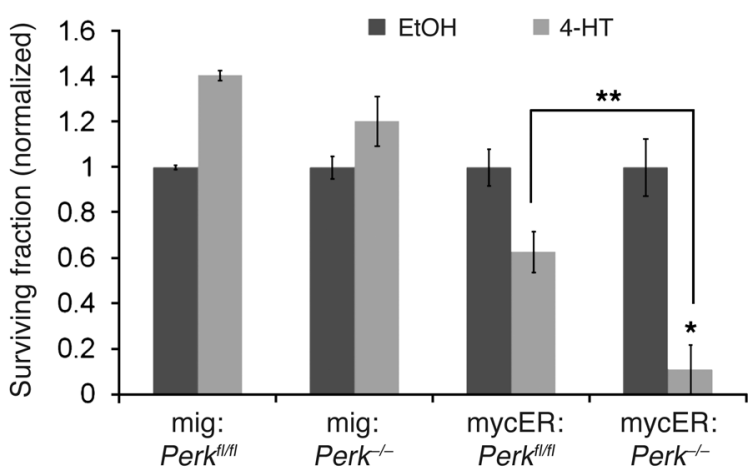

C $4-\mathrm{HT}$ Cl-PARP $\beta$-actin

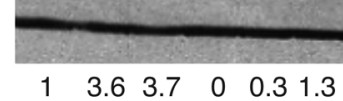

E

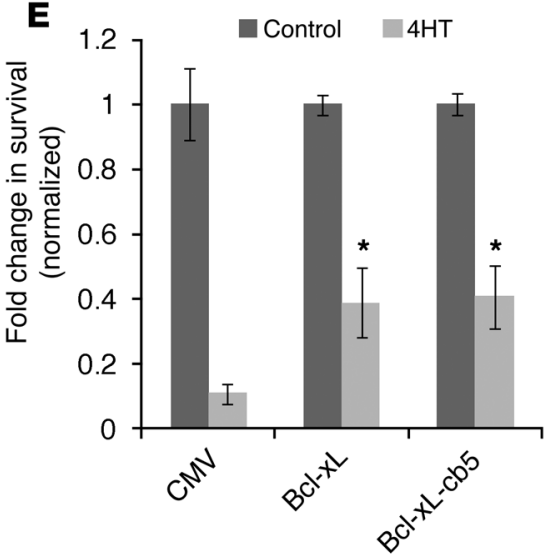

Figure 3

Loss of UPR signaling results in c-Myc-induced caspase-dependent apoptosis. (A) Perk ${ }^{f l / f l}$ or Perk ${ }^{-1-}$ MEFs infected with control (mig) or mycERexpressing retroviruses were treated with $\mathrm{EtOH}$ or $4-\mathrm{HT}$, and clonogenic survival was assayed (left panel); colonies were counted, and the surviving fraction is presented (right panel) normalized to each untreated control (error bars represent SEM; $n=3 ;{ }^{* *} P<0.0001,{ }^{*} P<0.0003$ ). (B) MEFs were treated with 4-HT for 24 hours, followed by immunoblotting for cleaved PARP (c-PARP). (C) mycER:Perk ${ }^{-1-}$ MEFs were treated with 4-HT in the presence or absence of 4-PBA (5 mM), and immunoblotting was performed for cleaved-PARP. (D) S51A-elF2 $\alpha$ knock-in MEFs infected with control (mig) or mycER-expressing retroviruses were treated with ethanol or 4-HT, and survival was assayed (compared with WT MEFs expressing mycER; ${ }^{* \star} P<0.00002,{ }^{*} P<0.002$, Student's 2-tailed $t$ test). (E) mycER:Perk ${ }^{-1-}$ MEFs were transfected with CMV control or $\mathrm{Bcl}-\mathrm{xL}$-expressing plasmids (WT or ER-targeted cb5), treated with 4-HT, cultured for 72 hours, and stained with crystal violet, and cell survival was quantified $\left({ }^{*} P<0.001\right.$, Student's 2 -tailed $t$ test).

corroborated the induction of apoptosis in PERK-deficient cells (Supplemental Figure 4, A and B). Furthermore, treatment of PERKdeficient cells with the chemical chaperone 4-PBA reduced the cytotoxic effect of c-Myc activation (Figure 3C), suggesting that c-Mycinduced cell death in the absence of PERK is dependent upon the presence of misfolded proteins. In order to determine whether eIF2 $\alpha$ phosphorylation and subsequent decreased translation are required for cell survival in PERK-proficient cells, we employed MEFs with an eIF $2 \alpha$ S51A knock-in mutation rendering the protein incapable of phosphorylation, thereby preventing UPR-induced downregulation of translation. As in PERK-deficient cells, Myc activation in S51A eIF2 $\alpha$ knock-in MEFs resulted in significantly more cell death relative to wild-type cells (Figure 3D and Supplemental Figure 4C).
To further characterize the c-Myc-induced apoptosis, we transiently overexpressed wild-type Bcl-xL or ER-targeted Bcl-xL-cb5 in mycER:Perk- MEFs and measured their response to Myc activation. Both wild-type Bcl-xL and ER-targeted Bcl-xL significantly rescued the PERK-deficient cells from Myc-induced apoptosis (Figure 3E and Supplemental Figure 4D). We also employed the pan-caspase inhibitory peptide Q-VD-OPH. The addition of Q-VD-OPH prevented c-Myc-induced PARP cleavage and rescued cell survival in mycER:Perk ${ }^{-1}$ MEFs treated with 4-HT and in cells treated with the proapoptotic agent staurosporine, whereas a negative control peptide had no cytoprotective effect (Supplemental Figure 4, E and F).

ER stress-induced apoptosis involves the release of intracellular $\mathrm{Ca}^{2+}$ from ER stores and subsequent cytochrome $c$ release from 
A

(8 h)
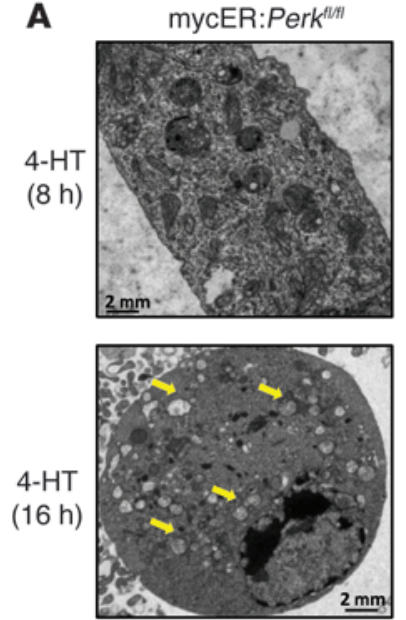

\section{mycER:Perk ${ }^{\prime}$}
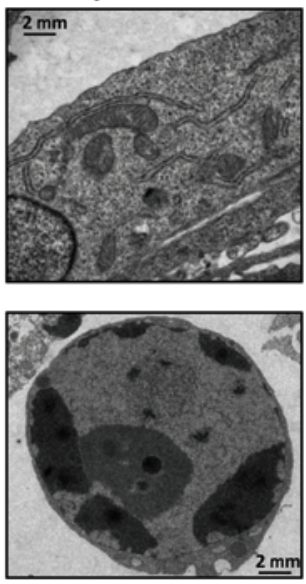

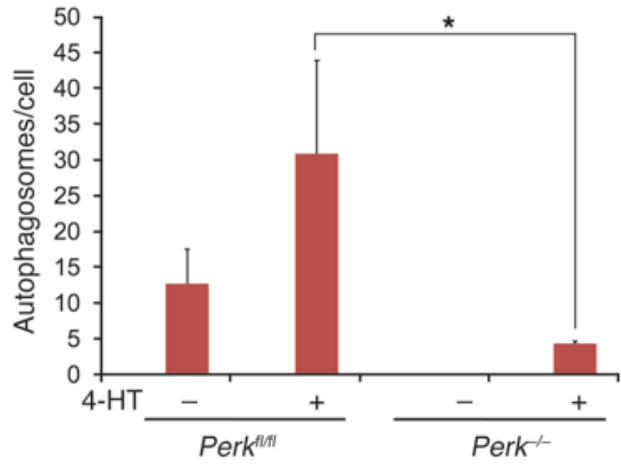
D \begin{tabular}{l} 
C-HT (h) \\
\cline { 2 - 2 }-81624
\end{tabular} p62 $\beta$-actin

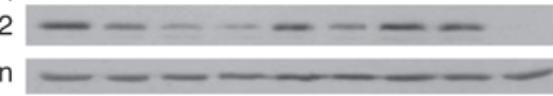

$\begin{array}{lllllllll}1.0 & 0.5 & 0.2 & 0.2 & 1.0 & 0.5 & 1.3 & 1.3\end{array}$

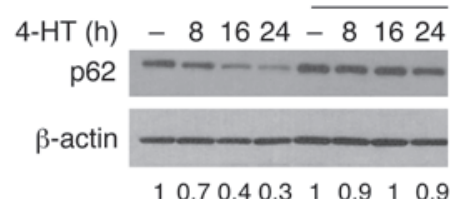

E

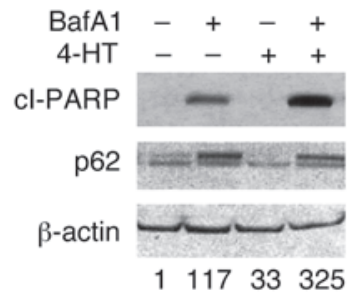

$\mathbf{F}$

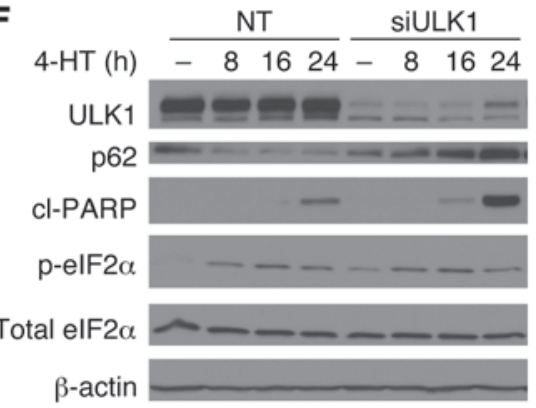

G

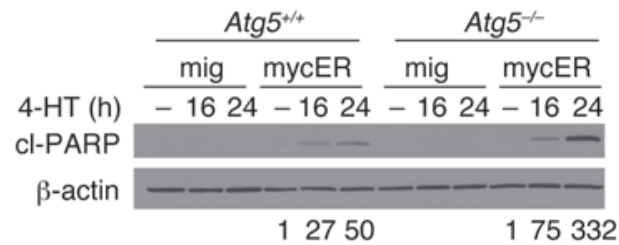

\section{Figure 4}

PERK-dependent autophagy promotes cell survival in cells expressing c-Myc. (A) MEFs were treated with 4-HT, then analyzed for autophagosome formation by electron microscopy (left panel; scale bars: $2 \mu \mathrm{m}$ ), and autophagosomes were quantified (right panel: 3-8 cells per treatment; ${ }^{*} P<0.05$, 1-tailed Student's $t$ test). (B) MEFs were treated with 4-HT and analyzed for processing of the autophagic marker LC3 with immunoblotting (blots are representative of 3 independent experiments; values represent the ratio of LC3II to LC3I and are shown as fold change relative control). (C) MEFs were treated with 4-HT, and immunoblotting was performed for degradation of p62 (Hanks solution used as positive control). (D) mycER:Perk ${ }^{f / f l}$ MEFs were treated with 4-HT in the presence or absence of 4-PBA, and immunoblotting was performed for p62. (E) mycER:Perk ${ }^{f l / f l}$ MEFs were treated with bafilomycin A1 (BafA1; $50 \mathrm{nM}$ ) and 4-HT, and cleaved PARP and p62 levels were analyzed. (F) mycER:Perk ${ }^{f / l f l} \mathrm{MEFs}$ were transfected with non-targeting (NT) or ULK1 siRNA, treated with 4-HT, and analyzed for autophagy and apoptosis. (G) Atg5 $5^{+/+}$and Atg5 $5^{-/-}$ MEFs were infected with control (mig) or mycER retrovirus and treated with 4-HT to detect cleaved PARP ( $\beta$-actin was used as a loading control; representative of 2 independent experiments; values below blot represent total pixel intensity of cleaved PARP normalized to actin for each lane and are shown as fold change relative to no tetracycline control).

mitochondria (41). We measured intracellular $\mathrm{Ca}^{2+}$ release by fluorescence microscopy with the Fura-2AM dye and found a significant increase in free intracellular calcium in mycER:Perk ${ }^{-/}$ MEFs treated with 4-HT (Supplemental Figure 4G). As expected, thapsigargin induced a more robust release of calcium. To further test whether the mechanism of cell death induced by c-Myc in the absence of PERK was ER stress dependent, we employed the calcium chelator BAPTA-AM to block the activity of released calcium. Whereas treatment of mycER:Perk ${ }^{-/-}$MEFs with 4-HT resulted in the cleavage of PARP, the presence of BAPTA-AM significantly reduced PARP cleavage (Supplemental Figure $4 \mathrm{H}$ ). Taken together, our results suggest that $\mathrm{c}-\mathrm{Myc}$ induces the accumulation of mis- folded proteins, which in the absence of PERK results in unmanageable ER stress, the subsequent release of calcium, and the initiation of caspase-dependent apoptosis.

c-Myc induces PERK-dependent cytoprotective autophagy. ER stressinduced autophagy was originally described in yeast, where activation of the UPR results in ER expansion and the formation of autophagosomes with ER-derived membranes (42). Moreover, UPR-induced activation of ATF4 has been shown to upregulate the autophagy genes ATG5 and MAP1LC3B to promote cell survival during acute hypoxic stress $(10,43)$. Additionally, autophagy has been shown to promote the survival of tumor cells in a mouse model of Myc-induced lymphomas (19). Therefore, we examined 
A

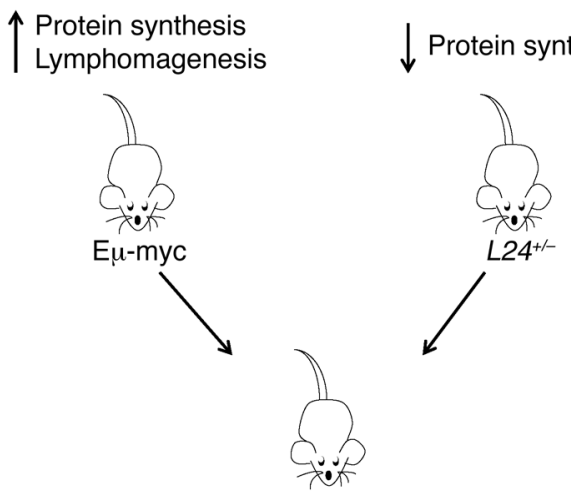

Eu-myc; L24+-

$\downarrow$ Near-WT protein synthesis

$\downarrow$ Lymphomagenesis

E

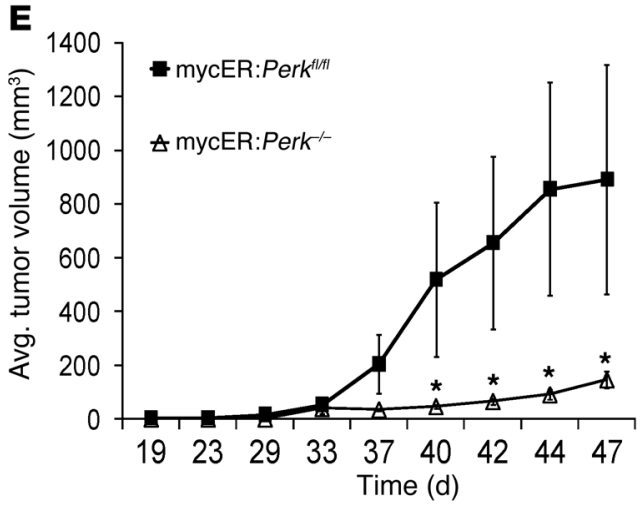

F

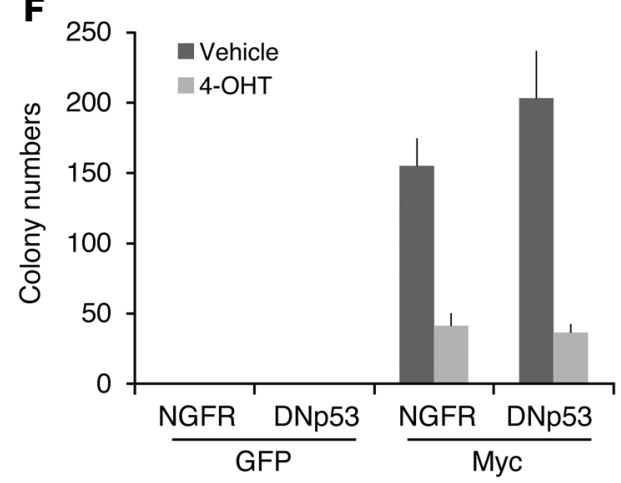

B

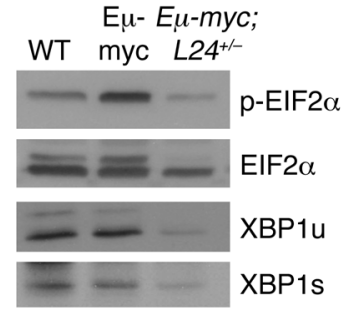

D

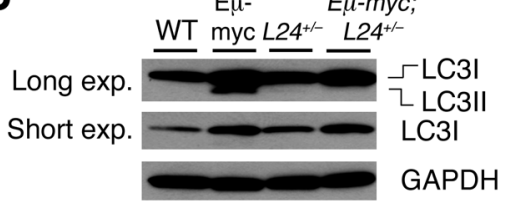

C

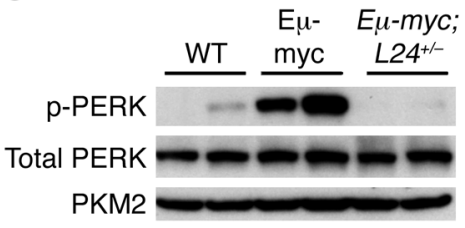

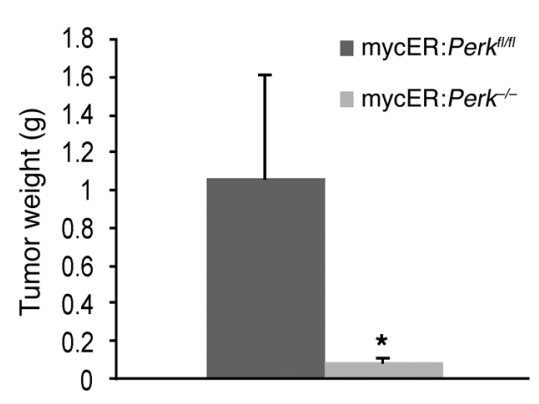

G

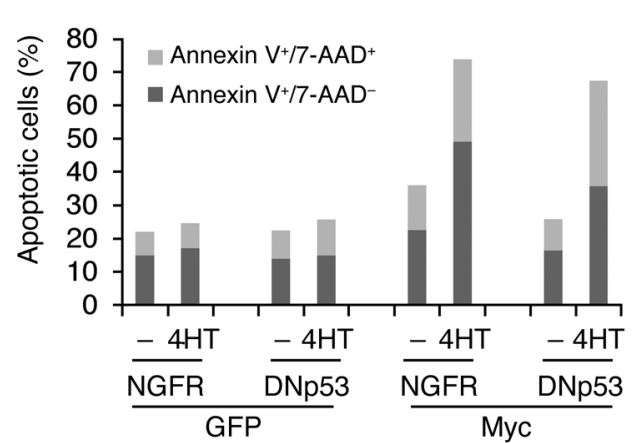

Figure 5

In vivo activation of the UPR in E $\mu$-myc transgenic mice and Myc-expressing murine HPCs. (A) Breeding scheme for the generation of $E \mu-m y c /+; L 24^{+/-}$double transgenic mice. (B) B lymphocytes from the spleens of WT, E $\mu-m y c /+$, and $E \mu-m y c /+; L 24^{+/-}$transgenic mice were isolated, and immunoblot analysis was performed to detect p-elF2 $\alpha$ (S51), total elF2 $\alpha$, and XBP1s (XBP1u, unspliced XBP1). (C) B lymphocytes from WT, E $\mu-m y c$, and E $\mu-m y c /+; L 24^{+/-}$mice were isolated, and immunoblot analysis was performed to detect total and phosphorylated PERK (lymphocytes from 2 mice per strain). (D) B lymphocytes from WT, E $\mu$-myc, and $E \mu-m y c /+; L 24^{+/-}$mice were analyzed for LC3II processing, indicative of autophagy. exp, exposure. (E) RasV12-transformed MEFs $\left(1 \times 10^{6}\right)$ were injected subcutaneously into the flanks of immunodeficient mice, and C-Myc was activated by intraperitoneal injection of tamoxifen every 2 days for the duration of the experiment. Tumor growth (left panel) and final tumor weight (right panel) measurements are shown ( $n=10$ tumors per group; ${ }^{*} P<0.04$, Student's 1-tailed $t$ test). (F and G) HPCs from Perk ${ }^{t / f f}$; CreER mice were isolated, infected to overexpress Myc and DN-p53, and treated with 4-HT to excise PERK; colony formation was assessed by culture in methylcellulose $(\mathbf{F})$, and cell death was measured by Annexin $V$ and 7-AAD staining $(\mathbf{G})$.

autophagy as a potential contributor to PERK-mediated cell survival. Electron microscopy revealed the presence of double-membrane vesicles, indicative of autophagosome formation in mycER:Perk $f^{l / f l}$ MEFs treated with 4-HT (Figure 4A). The mycER:Perk ${ }^{-/-}$MEFs lacked significant vesicle formation but demonstrated evidence of apoptosis, such as condensed chromatin in the nuclei. In addition to performing electron microscopy, we analyzed two markers of autophagy, LC3 and p62. The presence of acidic vesicles associated with autophagy results in the processing of LC3I (into LC3II) and the rapid degradation of the $\mathrm{p} 62$ protein. Treatment 
research article
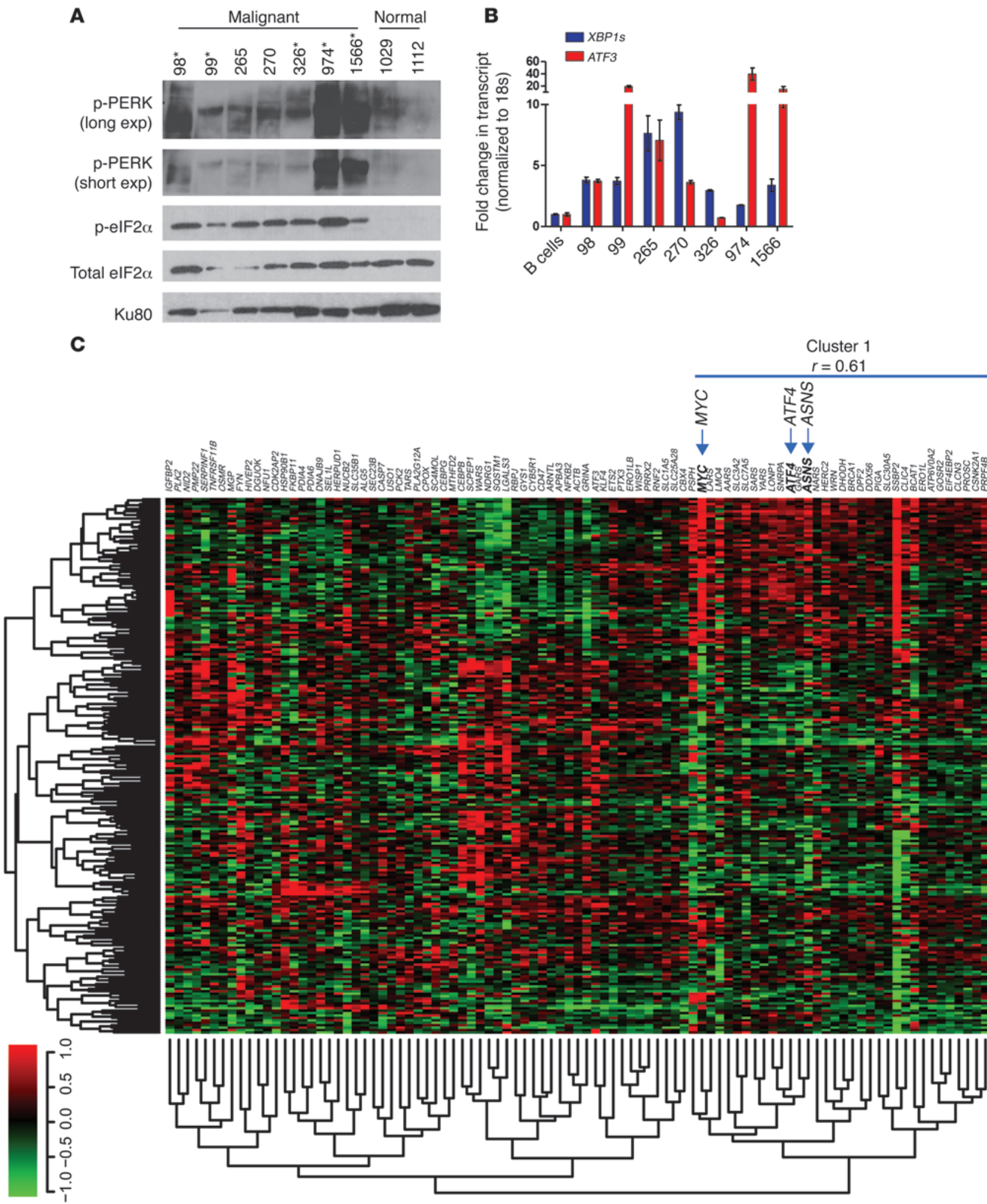

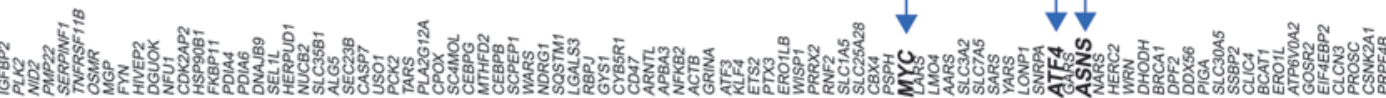
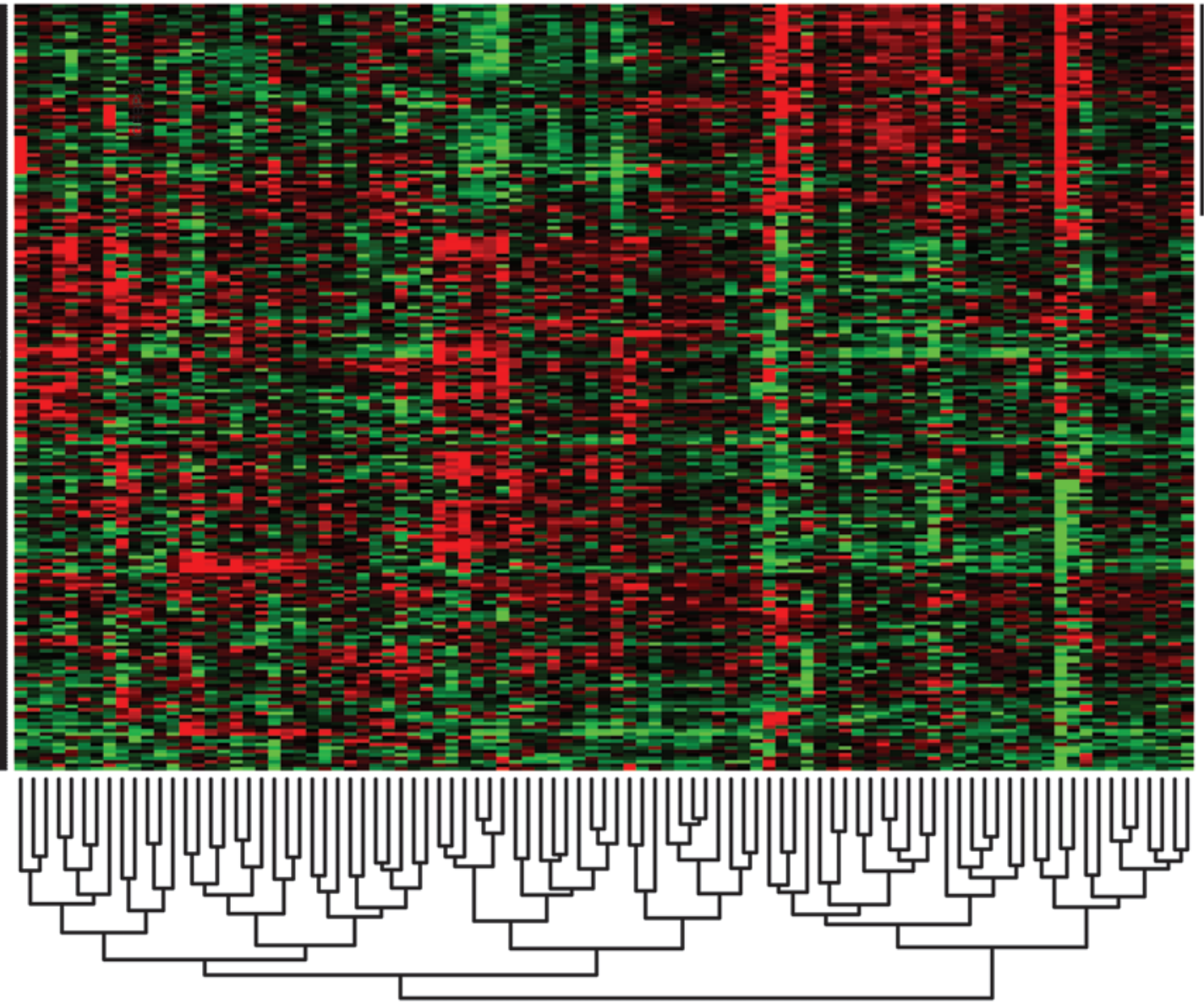


\section{Figure 6}

UPR activation in human lymphomas. (A) Primary human B cells, obtained from lymphoma patients and normal donors, were analyzed for UPR activation by p-elF2 $\alpha$ and p-PERK (Ser713) levels (*samples with Myc translocation confirmed by FISH analysis [note: Myc translocation was confirmed for patient 1566, who was diagnosed with AML]; all other samples were confirmed for Burkitt's lymphoma morphology and are presumed to contain Myc translocations). (B) Primary human $B$ cell samples were analyzed for downstream UPR activation by qPCR of XBP1s and ATF3 (average of 3 independent qPCR reactions). (C) An ER stress response signature clusters c-Myc-overexpressing B cell lymphomas. Raw data were downloaded from the NCBI GEO repository (GSE4475). The genes listed were derived from an ER stress response signature defined using ER stressors and genetic knockouts by Harding et al. (49). Normalized probe signals for the genes listed were clustered using a Pearson complete correlation coefficient, with a significance threshold for each hierarchical subcluster set at $P<0.05$. Cases annotated as Ig-Myc were defined as such by fluorescence in situ hybridization in the original expression array study. Expression signals are depicted using pseudocoloring, in which expression for each gene is shown as high (red) or low (green).

with thapsigargin resulted in PERK-dependent upregulation of LC3II levels in the mycER MEFs (Supplemental Figure 5A). Similar to thapsigargin-induced ER stress, c-Myc expression resulted in increased LC3II processing by both static (Figure 4B) and flux (Supplemental Figure 5B) assays, which was attenuated in Perk ${ }^{-/-}$MEFs. Additionally, p62 was efficiently degraded in the presence of c-Myc in a PERK-dependent manner (Figure 4C). In mycER:Perk ${ }^{-/-}$MEFs, we reproducibly observed a marginal, but temporary decrease in p62 levels at 8 hours following 4-HT treatment, suggesting that unsustained autophagic flux is induced in the absence of PERK signaling, further corroborated by the flux assay measuring LC3II (Supplemental Figure 5B). Starvation-induced autophagy in the mycER:Perk ${ }^{-/}$MEFs indicated that PERK-deficient cells are capable of inducing autophagy by means other than c-Myc expression. The degradation of $\mathrm{p} 62$ was dependent on the presence of misfolded proteins, as treatment of cells with 4-PBA rescued c-Myc-dependent p62 degradation (Figure 4D). c-Myc-induced autophagy was also apparent in P493-6 cells, as measured by LysoTracker staining (Supplemental Figure 5C). Collectively, these results indicate that autophagy is activated by c-Myc in a PERK-dependent manner.

To determine whether autophagy induction actively protects cells from c-Myc-induced apoptosis, we blocked autophagy in mycER:Perkflfl MEFs by treatment with bafilomycin A1 and measured cleaved PARP and p62 levels following induction of c-Myc expression. c-Myc-induced PARP cleavage was augmented in cells treated with bafilomycin A1, compared with vehicle-treated cells (Figure 4E). Increased cell death corresponds with increased p62, indicative of incomplete autophagy. We also observed a dosedependent effect of bafilomycin A1 on PARP cleavage in response to Myc activation (Supplemental Figure 5D). Spautin-1, a specific autophagy inhibitor, promotes the degradation of the Vps34Beclin 1 complex and prevents the initiation of autophagy and elongation of the autophagosome membrane (44). Spautin-1 treatment inhibited the accumulation of LC3II and, similar to bafilomycin A1 treatment, resulted in increased PARP cleavage in mycER:Perk $f^{l / f l}$ MEFs upon Myc activation (Supplemental Figure 5, E and F).

ULK1, a kinase involved in autophagy initiation, and Atg5, a member of the E3 ligase complex, are both required for complete autophagic flux $(45,46)$. To analyze the role of autophagy geneti- cally, we measured cleaved PARP levels following induction of c-Myc expression in mycER:Perkfl/fl cells transfected with siRNA targeting ULK1 and in $A \operatorname{tg} 5^{+/+}$and Atg $5^{-/-}$MEFs infected with mycER-expressing virus. c-Myc-induced PARP cleavage was increased in the absence of ULK1 or Atg5, compared with non-targeting siRNA or $\mathrm{Atg}^{+/+} \mathrm{MEFs}$, respectively (Figure 4, F and G). In addition to increased apoptosis, clonogenic survival was significantly reduced in Atg5-deficient MEFs treated with 4-HT compared with Atg5-proficient cells (Supplemental Figure 5G). Collectively, these results indicate that autophagy is activated in a PERK-dependent manner and promotes cell survival during c-Myc-induced activation of the UPR in vitro.

Ribosomal L24 heterozygosity rescues $c-M y c-i n d u c e d ~ P E R K$ activation and autophagy in Eu-myc lymphocytes. To test our findings in a physiological model of lymphomagenesis, we examined lymphocytes from the spleens of E $\mu$-myc and wild-type mice for activation of the UPR prior to tumor formation. The E $\mu$-myc transgenic mouse is an important model for Myc-induced tumor development in vivo. In E $\mu$-myc mice, c-Myc transgene expression is driven by the immunoglobulin transcriptional enhancer $E \mu$, and the mice develop B cell lymphomas and lymphoid leukemias (47), consistent with the fact that Myc is deregulated in 100\% of Burkitt's lymphoma in humans. Premalignant E $\mu$-myc lymphocytes from 5-week-old mice showed a substantial increase in UPR activation as measured by p-PERK, p-eIF2 $\alpha$, and XBP1s levels compared with wild-type littermate controls (Figure 5, B and C). We next assessed whether Myc-dependent increases in protein synthesis that augment the protein load of the ER may underlie UPR activation. We utilized a mouse minute mutant that lacks one copy of ribosomal protein L24 to restore Myc-dependent increases in protein synthesis to wild-type levels (ref. 32 and Figure 5A). Importantly, we have previously shown that ribosomal protein haploinsufficiency markedly attenuates E $\mu$-myc-driven lymphoma (32). Splenic lymphocytes from $E \mu-m y c /+; L 24^{+/-}$transgenic mice were analyzed for UPR activation prior to tumor formation (Figure 5, B and C) and were found to have near wild-type levels of p-PERK, p-eIF $2 \alpha$, and XBP1s. Additionally, the appearance of LC3II processing in lymphocytes from E $\mu$-myc mice confirms the induction of Myc-associated autophagy in vivo, which is prevented in double transgenic Eu-myc/+;L24+/- mice (Figure 5D).

Ablation of PERK leads to apoptosis of Myc-transformed primary cells and inhibition of tumor growth in vivo. We examined the effects of PERK status on the growth of c-Myc-driven Perk ${ }^{f l / f l}$ and Perk ${ }^{-/-}$MEFs in an allograft model. RasV12-transformed MEFs were injected subcutaneously in athymic mice, and the mice were treated with tamoxifen to induce c-Myc throughout the course of the experiment. Abrogation of PERK resulted in a nearly complete inhibition of c-Myc-dependent tumor growth (Figure 5E). These results strongly suggest that restraining the ability of c-Myc to increase protein synthesis (L24 heterozygosity) or attenuating Myc-induced UPR signaling (Perk $\left.{ }^{-/}\right)$disrupts c-Myc-associated ER stress, UPR activation, and autophagy in vivo and inhibits tumor growth.

To further test the role of PERK in Myc-induced transformation, we obtained hematopoietic progenitor cells (HPCs) from Perkflffl: CreER mice, treatment of which with 4-HT induces Cre recombinase activity resulting in PERK excision (Supplemental Figure 5I). Transformation of these cells with viruses overexpressing mycGFP and dominant-negative p53 results in colony formation in methylcellulose (Figure 5F) with minimal apoptosis, as measured by Annexin V-PE/7-AAD staining (Figure 5G). PERK excision via 
treatment with 4-HT resulted in significantly decreased colony formation and increased apoptosis (Figure 5, F and G). Therefore, several aspects of c-Myc-driven tumorigenesis are dependent on intact UPR signaling, suggesting a cytoprotective effect for UPR activation and providing a therapeutic context for the inhibition of PERK in c-Myc-dependent tumors.

UPR signaling is active in Myc-transformed human lymphomas. To interrogate the clinical relevance of these findings, we also analyzed UPR activation in primary human B cells isolated from patients with Burkitt's lymphoma. Nearly every case of Burkitt's lymphoma involves the translocation of the MYC proto-oncogene to the enhancer region of the heavy or light chain immunoglobulin locus, resulting in the constitutive overexpression of c-Myc in B lymphocytes (47). We found increased p-eIF2 $\alpha$ and p-PERK levels in all 7 malignant samples, compared with the nearly undetectable levels observed in B cells obtained from healthy donors (Figure 6A). Additionally, many of the malignant B cell samples had increased levels of XBP1s transcript and/or the ATF4 target ATF3 compared with normal B cells (Figure 6B).

Recently, promising results have come from a strategy of inhibiting c-Myc-dependent transcription of MYC with JQ1, a novel selective inhibitor of the c-MYC transcriptome (48). JQ1 is a small molecule BET bromodomain inhibitor that prevents c-Myc regulatory proteins from aiding in MYC transcription, effectively downregulating Myc transcriptional targets, and subsequently the levels of c-Myc protein itself in a range of disease models (48). We performed meta-analysis on genomic array datasets obtained from Raji human Burkitt's lymphoma cells treated with JQ1 or an inactive enantiomer. Specifically, the UPR gene signature (consisting of c-MYC, DDIT3 [CHOP], ATF3, and XBP1 mRNAs; ref. 49) is downregulated upon treatment with JQ1, but not with the enantiomer (Supplemental Figure 6).

To more extensively explore the association between c-Myc status and the expression of ER stress-regulated genes in clinical samples, we analyzed the Hummel dataset (50), for which c-Myc status was established by both fluorescence in situ hybridization and expression array profiling (GEO GSE4475). The UPR signature was generated using data from a study by Harding et al. (49), which identified genes that were responsive to ER stress treatments including tunicamycin in an ATF4- and PERK-dependent manner. We used this UPR signature to run a gene-level interpretation of the Hummel data and clinical dataset and generate data tables containing the log fold signals for the genes across all samples. These data were then subjected to a Pearson complete correlation analysis for hierarchical clustering and to annotate the resultant heatmap (Figure 6C). The Ig-Myc annotation is derived from the clinical annotation of tumor biopsy specimens in which myc was fused to $\operatorname{IgH}$, IgK, or IgL, referred to as "Ig-Myc lymphomas" in ref. 50. As shown in Figure 6C, Ig-Myc status closely correlates with increased c-Myc expression in the array data. The demonstrated activation of the UPR in human lymphoma samples together with the bromodomain dependency of UPR effector levels in the study by Mertz and colleagues (51) highlight the clinical relevance of our findings and strengthen the potential for UPR targeting as an alternative therapeutic strategy in Myc-driven malignancies.

\section{Discussion}

Tumor microenvironmental versus cell-autonomous activation of the UPR. Previous studies, including those of our group, have shown that the PERK-dependent and Ire1-dependent arms of the UPR are acti- vated in animal and human tumors, particularly in areas of hypoxia, and contribute to resistance of tumor cells to hypoxic stress and nutrient stress. Ablation of PERK, ATF4, IRE1, and XBP1 functions led to attenuation of tumor growth due to decreased resistance to these stresses, but also to decreases in the levels of angiogenesis $(8,52)$. These findings were later extrapolated to tumor models in transgenic mice, where PERK ablation led to the inhibition of growth rates in breast carcinoma and insulinoma $(12,13)$. However, it remained unclear whether cell-autonomous stresses such as those elicited by oncogenic activation could activate the UPR and what the functional consequences of such activation might be. Here, we describe a novel cell-autonomous mode of activation of the UPR in response to increased activity of the proto-oncogene Myc. Our findings indicate that PERK and subsequent eIF $2 \alpha$ phosphorylation act as a "molecular switch" that ultimately promotes transformation and overall tumorigenicity elicited by Myc. Under this prism, oncogenic activation of PERK could reflect a broader reliance of the transformed state on UPR than that imposed in response to non-cell-autonomous stresses, such as hypoxia and nutrient deprivation.

Previously, mutant H-Ras was shown to induce UPR activation and cellular senescence in a premalignant model of melanoma (53). In agreement with our work demonstrating a requirement of PERK for cell survival in the setting of oncogenic Myc activation, Denoyelle et al. showed that sustained inactivation of IRE1, ATF6, XBP1, or ATF-4 in the presence of HRAS ${ }^{\mathrm{N} 12}$ led to an increase in cell death. Loss of the tumor suppressor complex proteins TSC1 and TSC2 has also been shown to activate the UPR in a cell-autonomous manner (14). Thus, it is likely that ER stress signaling and UPR activation constitute a common cytoprotective mechanism to cope with hyperactivation of oncogenes or loss of tumor suppressors.

Antiapoptotic role of the PERK pathway: induction of autophagy and regulation of $E R$ calcium release. If the $\mathrm{UPR}$ is activated by c-Myc, what is its role in the context of transformation by Myc? It is now well established that activation of the UPR can have both pro-survival and pro-cell death consequences, depending on the cellular context, severity of stress, etc. Induction of ER stress-dependent apoptosis occurs after prolonged and/or severe ER stress caused by treatment with cytotoxic agents $(54,55)$. Conversely, due to their inability to downregulate protein synthesis, cells with a compromised UPR, such as those with abrogated PERK/eIF2 $\alpha$ or IRE1/ XBP1 signaling, are substantially more sensitive to ER-induced cell death than wild-type cells, presumably due to the continuous accumulation of misfolded proteins in the ER, a process termed proteotoxicity $(54,56)$. The mechanisms for ER-induced apoptosis are not completely understood, but there is strong evidence that $\mathrm{Ca}^{2+}$ release from ER stores induces cytochrome $c$ release from the mitochondria $(57,58)$. This leads to the subsequent activation of caspase-3, -4 , and -7 in human cells and of caspase-12 in rodents, which mediate apoptosis $(59,60)$. In our studies, we observed a significant increase in $\mathrm{Ca}^{2+}$ release from $\mathrm{Perk}^{-/-}$cells following Myc upregulation, and importantly, the calcium chelator BAPTA-AM rescued a significant portion of Myc-induced apoptosis in these MEFs. The $\mathrm{Ca}^{2+}$ release was not as acute as that observed following treatment with the SERCA inhibitor thapsigargin, and this likely reflects the fact that the effect is more gradual, as misfolded proteins accumulate in the ER. The incomplete rescue achieved by calcium chelation suggests that additional mechanisms are contributing to cytotoxicity by c-Myc in the absence of PERK. 


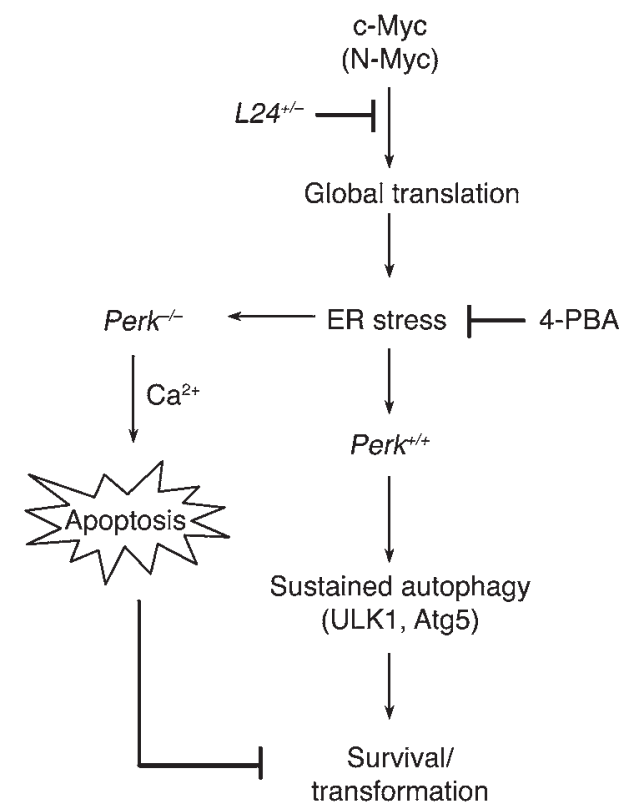

Figure 7

Model of the role of UPR activation in cytoprotection during Mycdependent transformation. c-Myc activation increases protein synthesis, resulting in UPR activation. This is attenuated by genetically reducing protein synthesis (L24 mouse minute) or pharmacologically increasing chaperone activity (4-PBA). In the presence of PERK, cytoprotective autophagy (LC3 processing, p62 degradation) is induced and is required for cell survival (ULK1 and Atg5 dependence). Loss of PERK results in significantly increased apoptosis, primarily through increase $\mathrm{Ca}^{2+}$ release form the ER and lack of autophagy.

Autophagy is another cytoprotective process that has been linked to activation of the UPR and particularly the PERK/eIF2 $\alpha$ pathway (a process termed ER-phagy) (42). However, it has also been postulated that under certain physiological conditions and in response to specific treatments, autophagy leads to enhanced clonogenic cell death $(17,61)$. Sustained autophagy without a balancing synthesis of new materials would result in detrimental self-cannibalism beyond the possibility of cellular recovery. Previous work in yeast and mammalian cells has established that ER stress can activate autophagy. Rzymski et al. have also reported that tumor hypoxia can enhance autophagic flux and thereby promote resistance to hypoxia-induced apoptosis both in vitro and in vivo $(10,62)$. It has been proposed that stressors such as nutrient deprivation result in the dissociation of Beclin 1 from Bcl-2, thereby counteracting apoptotic mechanisms, and that, importantly, the inhibition of autophagy makes cells more susceptible to stress-induced apoptosis (63). Indeed, our results show that PERK-mediated processing of LC3 and downregulation of p62, both markers of autophagy, occur following Myc activation. Interestingly, Maclean et al. have reported that in the E $\mu$-myc mouse model, an inhibitor of autophagic flux, chloroquine, increased clonogenic death of Myc-dependent lymphoma cells and impaired lymphoma development in a Burkitt's lymphoma model, pointing toward a cytoprotective role of autophagy under c-Myc activation $(19,64)$. Moreover, we found evidence of LC3 processing in B cells from $\mathrm{E} \mu$-myc mice but not from wild-type or double transgenic $E \mu-m y c /+; L 24^{+/-}$mice, indicating that relieving ER stress by downregulating of protein load also alleviates autophagic induction.
A link between Myc-induced translation, UPR activation, and transformation. It has been established that Myc-induced transformation requires the cooperation of pro-survival pathways for full transformation and tumorigenesis. It is likely that multiple such pathways with antiapoptotic activity may contribute to eventual transformation by Myc. Recently, one of our groups showed that protein synthesis is required for Myc-dependent tumorigenesis and optimal lymphoma progression (32). Importantly, this study demonstrated that restoration of $\mathrm{Myc}$-induced protein synthesis rates to wild-type levels led to an increase in apoptosis and reduced lymphomagenesis, thereby uncovering a link between translational control by Myc and cell survival. Here, we show that one of the mechanisms by which Myc-overexpressing cells attain a survival advantage is protein synthesis-dependent activation of the UPR (Figure 7). Indeed, we demonstrate the mechanistic link between Myc-driven increases in protein synthesis and UPR activation both pharmacologically and genetically (Figure 5). The absence of PERK and eIF2 $\alpha$ phosphorylation in Myc-overexpressing MEFs, and the absence of PERK from Myc-overexpressing bone marrow hematopoietic progenitor cells results in massive apoptosis and reduced clonogenicity (MEFs and HPCs) and reduced tumor growth (MEFs) when Myc is hyperactivated. Therefore, we have identified PERK as a sensor of the translational status of the cell and regulator of cell survival in the context of Myc hyperactivation. Our findings support the use of PERK inhibitors as an approach to target tumors driven by oncogenic Myc.

Collectively, our findings support a model (Figure 7) in which activation of autophagy in a PERK-dependent manner and amelioration of $\mathrm{Ca}^{2+}$ release by PERK contribute to increased resistance of transformed cells to c-Myc upregulation and increased ER load. Oncogenic activation of the UPR downstream of MYC amplification provides one possible escape mechanism for oncogeneinduced stress that is required for cellular transformation and highlights a novel means of treating malignancies characterized by MYC translocations and amplifications.

\section{Methods}

\section{Cell lines, plasmids, and chemicals}

Cell lines were maintained in DMEM (Perk ${ }^{l / f l}$ and Perk ${ }^{-/-}$MEFs, Atg5 $5^{+/+}$and Atg 5 ${ }^{-1-}$ MEFs) or RPMI-1640 (P493-6, SHEP N-MycER, and SHEP Tet-21) supplemented with $10 \%$ fetal bovine serum and penicillin/streptomycin and cultured in $5 \% \mathrm{CO}_{2}$ at $37^{\circ} \mathrm{C}$. Cell lines were provided by D. Cavener (Penn State University, State College, Pennsylvania, USA) (Perk ${ }^{f / f l}$ and Perk ${ }^{-/}$MEFs) (65), D. Eick (Center of Integrated Protein Science [CIPSM], Helmholtz Center Munich, Munich, Germany) (P493-6) (66), C. Beltinger (University Children's Hospital, Ulm, Germany) (N-MycER SHEP) (36), J. Shohet (Baylor College of Medicine, Houston, Texas, USA) and M. Schwab (German Cancer Research Center, University of Heidelberg, Heidelberg, Germnay) (SHEP Tet-21) (37), and C. Thompson and T. Lindsten (Memorial Sloan-Kettering Cancer Center, New York, New York, USA) $\left(\right.$ Atg $5^{+/+}$ and Atg5 $5^{-1-}$ MEFs) (67). The MEFs were infected with retroviruses expressing either the mycER construct or control empty vector (mig), and stable clones were selected with puromycin. PERK was excised from the MEFs using an adenovirus expressing Cre recombinase, and clones were screened for loss of PERK. mycER activation was performed by treatment with 4-HT at $250 \mathrm{nM}$ (vehicle was ethanol). The following reagents were used (purchased from Sigma-Aldrich unless otherwise noted): 4-HT, tamoxifen, tetracycline, thapsigargin, tunicamycin, staurosporine (MP Biomedicals), 4-PBA (Calbiochem), Q-VD-OPH (BioVision), BAPTA-AM, E64d, pepstatin A, bafilomycin A1, and Spautin-1 (Cellagen Technology). 


\section{Immunoblotting}

Protein was harvested from cell lines as previously described (68). Immunoblotting was performed on extracts as previously described (68), with the exception that proteins were transferred to polyvinylidene fluoride membranes. See Supplemental Methods for details about the specific antibodies used.

\section{Flow cytometry and immunofluorescence}

ER-Tracker and LysoTracker studies. P493-6 cells were treated, collected, and resuspended $\left(1 \times 10^{6}\right.$ cells $\left./ \mathrm{ml}\right)$ in complete RPMI-1640 medium. ER-Tracker ( $500 \mathrm{nM}$ for 30 minutes) and LysoTracker ( $100 \mathrm{nM}$ for 45 minutes) dyes (Invitrogen) were added to the resuspended cells in individual reactions at $37^{\circ} \mathrm{C}$. Cells were then pelleted, resuspended in HBSS, and analyzed on a BD LSR II (BD Biosciences) flow cytometer at the University of Pennsylvania Flow Cytometry and Cell Sorting facility. Fluorophores were excited with a green 532-nm laser, and emission was captured with a 575/25 BP (LysoTracker) or a 610/20BP (ER-Tracker) and 600LP dichroic. Cell size was assessed based on forward scatter analysis. A similar ER-Tracker incubation was performed on attached cells for immunofluorescence images of ER. Counterstaining was performed with DAPI. Fluorescence intensity and area of the ER (red) and nucleus (blue) were quantified in binary format, values were corrected for background, volume was determined by multiplying area by intensity, and final ER volumes were divided by nucleus volumes for each cell.

Cell cycle and sub- $G_{1}$ analysis. Cells were harvested with trypsin, washed, resuspended in PBS (1\% FBS), and fixed with cold ethanol. Fixed cells were washed with PBS, treated with phosphate-citric acid buffer for 5 minutes, and resuspended in PI/RNase staining solution (PBS, RNase, and $50 \mu \mathrm{g} / \mathrm{ml}$ propidium iodide). Stained cells were stored for 30 minutes at room temperature in the dark and analyzed by a flow cytometry (FACSCalibur or LSR, CellQuest Pro; BD Biosciences).

\section{Electron microscopy}

Cells were cultured in 60-mm coated tissue dishes and treated with 4-HT (or ethanol control) at less than $70 \%$ confluency at the time of fixation. Cells were rinsed once with pre-warmed fixative, then for 60 minutes at room temperature, at which point cells were submitted to the University of Pennsylvania Biomedical Imaging Core/Electron Microscopy Resource Laboratory for analysis. At least 40 cells were analyzed from each treatment group, and representative images are shown.

\section{Clonogenic survival assay for $M E F$}

Clonogenic assays were performed as previously described (68). Briefly, cells were harvested, counted, plated at low density in triplicate, and treated with either vehicle (ethanol) or 4-HT (250 nM) for the duration of the experiment. Cells were cultured for 7-10 days or until viable colonies reached $>100$ cells. Colonies were stained with crystal violet $(0.4 \%$ crystal violet, $20 \%$ ethanol) and counted. Modified clonogenic assays were performed in 24-well plates and plated at a higher density. Quantitation of modified clonogenic assays involved the resolubilization of crystal violet in 33\% acetic acid to obtain absorbance readings at $540 \mathrm{~nm}$, as previously described (69). Colony counts were normalized to plating efficiency and represented as fraction surviving compared with control (ethanol). SEM values from triplicate plating represent at least 2 independent clonogenic assays.

\section{Image analysis}

To calculate the area or the ER versus nuclei, images were converted to binary format, and fluorescence intensity for the ER (ER-Tracker; red) and the nucleus (DAPI; blue) was calculated along with the corresponding areas. CTFI (corrected fluorescence intensity) was calculated by subtract- ing a blank measurement from the mean fluorescence of each cell. Densitometric volumes were then determined by multiplying area by intensity, for 8 control (EtOH-treated) cells and 8 Myc-induced (4-HT-treated) cells.

\section{Bone marrow transformation studies}

Constructs and retroviruses. MSCV-myc-IRES-GFP has been reported elsewhere (70). DNp53 was subcloned into the BglII/EcoRI-digested MSCVIRES-NGFR vector (gift from Warren Pear, University of Pennsylvania). Retrovirus was produced with the constructs above by individually cotransfecting them with Pcl-Eco retrovirus packaging vector into 293T cells using Lipofectamine reagent (Invitrogen). Retroviral supernatant was harvested 48 and 72 hours after transfection, filtered, and stored at $-80^{\circ} \mathrm{C}$. The titer of the infectious virus was determined by flow cytometry using NIH 3 T3 cells infected with serial dilutions of virus in the presence of $8 \mu \mathrm{g} / \mathrm{ml}$ Polybrene.

Transformation assay. Bone marrow cells from Perk $\ell^{l / f l} ;$ CreER mice treated with 5 -FU $(150 \mathrm{mg} / \mathrm{kg})$ were retrovirally transduced (in the presence of IL-3, IL-6, and SCF) with myc-GFP, DNp53-NGFR, or the vector controls as indicated. GFP/NGFR double-positive cells were sorted and replated weekly in methycellulose medium with 4-HT or ethanol vehicle. Colony numbers were counted after each replating, and colonies with $>50$ cells were scored and assessed for 3 rounds of serial replating.

Bone marrow apoptosis studies. Bone marrow cells were treated with 4-HT or vehicle for 4 days, transduced with the retroviruses as already described, and lysed with ACK buffer (BioWhittaker) and stained with APC-NGFR (BD Bioscience) 30 hours after transduction. The stained cells were further stained with Annexin V-PE/7-AAD according to the manufacturer's instructions (BD Biosciences).

\section{MEF tumor growth studies}

MEFs $\left(1 \times 10^{6}\right.$ cells) were injected subcutaneously in the flanks of athymic nude mice (Taconic) with a total of 10 tumors per group $(n=10)$. For tamoxifen treatment, the hormone powder (Sigma-Aldrich) was dissolved by sonication in peanut oil $(10 \mathrm{mg} / \mathrm{ml})$, and mice were injected every 2 days (i.p.) at the dose of $1 \mathrm{mg}$ per mouse. Tumor growth was measured with calipers. Tumor volume was calculated with the following equation: volume $=0.5\left(\right.$ length $\times$ width $\left.{ }^{2}\right)$.

Primary B cell isolation. E $\mu-m y c /+, E \mu-m y c /+; L 24^{+/-}$, and WT mice were sacrificed at 5 weeks of age, and the spleens were harvested. Splenic B cells were purified by using a B cell isolation kit (Miltenyi Biotec 130-090-862) in conjunction with an AutoMACS cell sorter per the manufacturer's protocol. Cell pellets were snap frozen in liquid nitrogen prior to RIPA lysis and immunoblot analysis, as previously described (32).

\section{Human tumor samples}

All human samples were obtained from patients presenting with acute myeloid leukemia. They were provided by the Stem Cell and Xenograft Core Facility at the University of Pennsylvania School of Medicine. Leukapheresis samples were frozen in fetal calf serum with $10 \%$ DMSO and stored in liquid nitrogen. French-American-British (FAB) or WHO classification and cytogenetics in AML samples were determined at the time of diagnosis by the Laboratory of Pathology and Medicine at the Hospital of the University of Pennsylvania. Protein and RNA from 7 malignant samples and 2 samples obtained from normal donors were harvested for immunoblot and qPCR analysis, as described in the Supplemental Methods.

\section{Statistics}

Where necessary, data were statistically analyzed to generate standard error values and determine the level of significance using the Student's $t$ test (1-tailed or 2 -tailed tests are denoted in the corresponding figure legends). Where appropriate, $P$ values are provided in the legends. Data represent mean values \pm SEM. 


\section{Study approval}

For human tissues, informed consent was obtained under a protocol reviewed and approved by the Institutional Review Board at the University of Pennsylvania (IRB protocol 703185). All animal experiments were approved by and performed according to University of Pennsylvania and UCSF IACUC regulations.

\section{Acknowledgments}

We thank Douglas Cavener for providing Perkflffl mice for the isolation of the MEFs and Craig Thompson and Tulia Lindsten for sharing the Atg $5^{+/+}$and $A \operatorname{tg} 5^{-/-}$MEFs. We also thank Carly Sayers for critical reading of the manuscript and Michael Dews and Elena Sotillo-Pineiro for technical guidance. D. Ruggero is a Leukemia and Lymphoma Society Scholar. This work is supported by NIH R01CA094214 and NIH R01CA139362 (to C. Koumenis); ACS 121364-PF-11-184-01-TBG (to J.T. Cunningham); NIH R01CA140456 (to D. Ruggero); R01CA122334 (to
A. Thomas-Tikhonenko); and by Commonwealth of Pennsylvania Universal Research Enhancement Program Funds and P01CA104838 to J. Alan Diehl.

Received for publication January 19, 2012, and accepted in revised form September 6, 2012.

Address correspondence to: Constantinos Koumenis, Department of Radiation Oncology, University of Pennsylvania Perelman School of Medicine, Translational Research Center, Room 8-087, 3400 Civic Center Blvd., Bldg 421, Philadelphia, Pennsylvania 19104-5156, USA. Phone: 215.898.0076; Fax: 215.756.6286; E-mail: koumenis@xrt.upenn.edu.

Lori S. Hart's present address is: Division of Oncology and Center for Childhood Cancer Research, Children's Hospital of Philadelphia, Philadelphia, Pennsylvania, USA.
1. Ron D, Walter P. Signal integration in the endoplasmic reticulum unfolded protein response. Nat Rev Mol Cell Biol. 2007;8(7):519-529.

2. Malhotra JD, Kaufman RJ. The endoplasmic reticulum and the unfolded protein response. Semin Cell Dev Biol. 2007;18(6):716-731.

3. Yoshida H, Matsui T, Yamamoto A, Okada T, Mori K. XBP1 mRNA is induced by ATF 6 and spliced by IRE1 in response to ER stress to produce a highly active transcription factor. Cell. 2001;107(7):881-891.

4. Bertolotti A, Zhang Y, Hendershot LM, Harding HP, Ron D. Dynamic interaction of $\mathrm{BiP}$ and ER stress transducers in the unfolded-protein response. Nat Cell Biol. 2000;2(6):326-332.

5. Haze K, Yoshida H, Yanagi H, Yura T, Mori K. Mammalian transcription factor ATF6 is synthesized as a transmembrane protein and activated by proteolysis in response to endoplasmic reticulum stress. Mol Biol Cell. 1999;10(11):3787-3799.

6. Krishnamoorthy T, Pavitt GD, Zhang F, Dever TE, Hinnebusch AG. Tight binding of the phosphorylated alpha subunit of initiation factor 2 (eIF2alpha) to the regulatory subunits of guanine nucleotide exchange factor eIF2B is required for inhibition of translation initiation. Mol Cell Biol. 2001;21(15):5018-5030.

7. Blais JD, et al. Activating transcription factor 4 is translationally regulated by hypoxic stress. Mol Cell Biol. 2004;24(17):7469-7482.

8. Bi M, et al. ER stress-regulated translation increases tolerance to extreme hypoxia and promotes tumor growth. EMBO J. 2005;24(19):3470-3481.

9. Romero-Ramirez L, et al. XBP1 is essential for survival under hypoxic conditions and is required for tumor growth. Cancer Res. 2004;64(17):5943-5947.

10. Rouschop KMA, et al. The unfolded protein response protects human tumor cells during hypoxia through regulation of the autophagy genes MAP1LC3B and ATG5. J Clin Invest. 2010; 120(1):127-141.

11. Fu Y, et al. Pten null prostate tumorigenesis and AKT activation are blocked by targeted knockout of ER chaperone GRP78/BiP in prostate epithelium. Proc Natl Acad Sci U S A. 2008;105(49):19444-19449.

12. Bobrovnikova-Marjon E, et al. PERK promotes cancer cell proliferation and tumor growth by limiting oxidative DNA damage. Oncogene. 2010;29(27):3881-3895.

13. Gupta S, McGrath B, Cavener DR. PERK regulates the proliferation and development of insulinsecreting beta-cell tumors in the endocrine pancreas of mice. PLoS ONE. 2009;4(11):e8008.

14. Ozcan U, et al. Loss of the tuberous sclerosis complex tumor suppressors triggers the unfolded protein response to regulate insulin signaling and apoptosis. Mol Cell. 2008;29(5):541-551.

15. Rabinowitz JD, White E. Autophagy and metabolism. Science. 2010;330(6009):1344-1348.

16. Hippert MM, O'Toole PS, Thorburn A. Autophagy in cancer: good, bad, or both? Cancer Res. 2006; 66(19):9349-9351.

17. Turcotte S, Chan DA, Sutphin PD, Hay MP, Denny WA, Giaccia AJ. A molecule targeting VHL-deficient renal cell carcinoma that induces autophagy. Cancer Cell. 2008;14(1):90-102.

18. White E, DiPaola RS. The double-edged sword of autophagy modulation in cancer. Clin Cancer Res. 2009;15(17):5308-5316.

19. Amaravadi RK, Thompson CB. The roles of therapy-induced autophagy and necrosis in cancer treatment. Clin Cancer Res. 2007;13(24):7271-7279.

20. Bernales S, Schuck S, Walter P. ER-phagy: selective autophagy of the endoplasmic reticulum. Autophagy. 2007;3(3):285-287.

21. Kouroku Y, et al. ER stress (PERK//eIF2[alpha] phosphorylation) mediates the polyglutamineinduced LC3 conversion, an essential step for autophagy formation. Cell Death Differ. 2007; $14(2): 230-239$.

22. He C, Klionsky DJ. Regulation mechanisms and signaling pathways of autophagy. Annu Rev Genet. 2009; 43:67-93.

23. Dang CV. Enigmatic MYC conducts an unfolding systems biology symphony. Genes Cancer. 2010; 1(6):526-531.

24. Green DR, Evan GI. A matter of life and death. Cancer Cell. 2002;1(1):19-30.

25. Hanahan D, Weinberg RA. The hallmarks of cancer. Cell. 2000;100(1):57-70.

26. Ruggero $\mathrm{D}$, et al. The translation factor eIF-4E promotes tumor formation and cooperates with c-Myc in lymphomagenesis. Nat Medicine. 2004;10(5):484-486.

27. Hemann MT, et al. Evasion of the p53 tumour surveillance network by tumour-derived MYC mutants. Nature. 2005;436(7052):807-811.

28. Pelengaris S, Khan M, Evan GI. Suppression of Myc-induced apoptosis in beta cells exposes multiple oncogenic properties of Myc and triggers carcinogenic progression. Cell. 2002;109(3):321-334.

29. Gao P, et al. c-Myc suppression of miR-23a/b enhances mitochondrial glutaminase expression and glutamine metabolism. Nature. 2009;458(7239):762-765.

30. Meyer N, Penn LZ. Reflecting on 25 years with MYC. Nat Rev Cancer. 2008;8(12):976-990.

31. Iritani BM, Eisenman RN. c-Myc enhances protein synthesis and cell size during B lymphocyte development. PNAS. 1999;96(23):13180-13185.

32. Barna M, et al. Suppression of Myc oncogenic activity by ribosomal protein haploinsufficiency. Nature. 2008;456(7224):971-975.
33. Chan JC, et al. AKT promotes rRNA synthesis and coorperates with c-MYC to stimulate ribosome biogenesis in cancer. Sci Signal. 2011;4(188):ra56.

34. Ruggero D. The role of Myc-induced protein synthesis in cancer. Cancer Res. 2009;69(23):8839-8843.

35. van Riggelen J, Yetil A, Felsher DW. MYC as a regulator of ribosome biogenesis and protein synthesis. Nat Rev Cancer. 2010;10(4):301-309.

36. Ushmorov A, Hogarty MD, Liu X, Knausz H, Debatin KM, Beltinger C. N-myc augments death and attenuates protective effects of $\mathrm{Bcl}-2$ in trophically stressed neuroblastoma cells. Oncogene. 2008; 27(24):3424-3434.

37. Lutz W, Stöhr M, Schürmann J, Wenzel A, Löhr A, Schwab M. Conditional expression of $\mathrm{N}$-myc in human neuroblastoma cells increases expression of alpha-prothymosin and ornithine decarboxylase and accelerates progression into S-phase early after mitogenic stimulation of quiescent cells. Oncogene. 1996;13(4):804-812.

38. Harding HP, et al. Regulated translation initiation controls stress-induced gene expression in mammalian cells. Mol Cell. 2000;6(5):1099-1108.

39. Vattem KM, Wek RC. Reinitiation involving upstream ORFs regulates ATF 4 mRNA translation in mammalian cells. PNAS. 2004;101(31):11269-11274.

40. Ozcan U, et al. Chemical chaperones reduce ER stress and restore glucose homeostasis in a mouse model of Type 2 diabetes. Science. 2006; 313(5790):1137-1140

41. Deniaud A, et al. Endoplasmic reticulum stress induces calcium-dependent permeability transition, mitochondrial outer membrane permeabilization and apoptosis. Oncogene. 2008;27(3):285-299.

42. Bernales S, McDonald KL, Walter P. Autophagy counterbalances endoplasmic reticulum expansion during the unfolded protein response. PLoS Biol. 2006;4(12):e423.

43. Sakaki K, Kaufman R. Regulation of ER stressinduced macroautophagy by protein kinase C. Autophagy. 2008;4(6):841-843.

44. Liu J, et al. Beclin1 Controls the levels of p53 by regulating the deubiquitination activity of USP10 and USP13. Cell. 2011;147(1):223-234.

45. Ganley IG, Lam DH, Wang J, Ding X, Chen S, Jiang X. ULK1.ATG13.FIP200 complex mediates mTOR signaling and is essential for autophagy. $J$ Biol Chem. 2009;284(18):12297-12305.

46. Mathew R, et al. Autophagy suppresses tumor progression by limiting chromosomal instability. Genes Dev. 2007;21(11):1367-1381.

47. Harris AW, Pinkert CA, Crawford M, Langdon WY, Brinster RL, Adams JM. The E-mu-myc transgenic mouse: a model for high-incidence spontaneous lymphoma and leukemia of early B cells.J Exp Med. 
1988;167(2):353-371.

48. Delmore JE, et al. BET bromodomain inhibition as a therapeutic strategy to target c-Myc. Cell. 2011; 146(6):904-917.

49. Harding HP, et al. An integrated stress response regulates amino acid metabolism and resistance to oxidative stress. Mol Cell. 2003;11(3):619-633.

50. Hummel M, et al. A biologic definition of burkitt's lymphoma from transcriptional and genomic profiling. N Engl J Med. 2006;354(23):2419-2430

51. Mertz JA, et al. Targeting MYC dependence in cancer by inhibiting BET bromodomains. Proc Nat Acad Sci U S A. 2011;108(40):16669-16674.

52. Blais JD, et al. Perk-dependent translational regulation promotes tumor cell adaptation and angiogenesis in response to hypoxic stress. Mol Cell Biol. 2006; 26(24):9517-9532.

53. Denoyelle C, et al. Anti-oncogenic role of the endoplasmic reticulum differentially activated by mutations in the MAPK pathway. Nat Cell Biol. 2006;8(10):1053-1063.

54. Koumenis C. ER stress, hypoxia tolerance and tumor progression. Curr Mol Med. 2006;6(1):55-69.

55. Ma Y, Hendershot LM. The role of the unfolded protein response in tumour development: friend or foe? Nat Rev Cancer. 2004:4(12):966-977.

56 . Ron D. Proteotoxicity in the endoplasmic reticu- lum: lessons from the Akita diabetic mouse. J Clin Invest. 2002;109(4):443-445.

57. Scorrano L, et al. BAX and BAK regulation of endoplasmic reticulum $\mathrm{Ca} 2+$ : a control point for apoptosis. Science. 2003;300(5616):135-139.

58. Pinton P, Giorgi C, Pandolfi PP. The role of PML in the control of apoptotic cell fate: a new key player at ER-mitochondria sites. Cell Death Differ. 2011; 18(9):1450-1456.

59. Boyce M, Yuan J. Cellular response to endoplasmic reticulum stress: a matter of life or death. Cell Death Differ. 2006;13(3):363-373.

60. Hetz C, Soto C. Stressing out the ER: a role of the unfolded protein response in prion-related disorders. Curr Mol Med. 2006;6(1):37-43.

61 Kondo Y, Kondo S. Autophagy and cancer therapy. Autophagy. 2006;2(2):85-90

62. Rzymski T, et al. Regulation of autophagy by ATF4 in response to severe hypoxia. Oncogene. 2010; 29(31):4424-4435.

63. Levine B, Sinha SC, Kroemer G. Bcl-2 family members: dual regulators of apoptosis and autophagy. Autophagy. 2008;4(5):600-606.

64. Maclean KH, Dorsey FC, Cleveland JL, Kastan MB. Targeting lysosomal degradation induces p53-dependent cell death and prevents cancer in mouse models of lymphomagenesis. J Clin Invest.
2008;118(1):79-88

65. Zhang $\mathrm{P}$, et al. The PERK eukaryotic initiation factor 2 alpha kinase is required for the development of the skeletal system, postnatal growth, and the function and viability of the pancreas. Mol Cell Biol. 2002;22(11):3864-3874.

66. Pajic A, et al. Cell cycle activation by c-myc in a Burkitt lymphoma model cell line. Int J Cancer. 2000; 87(6):787-793.

67. Cheong H, Lindsten T, Wu J, Lu C, Thompson CB. Ammonia-induced autophagy is independent of ULK1/ULK2 kinases. Proc Natl Acad Sci U S A. 2011; 108(27):11121-11126

68. Koumenis C, et al. Regulation of protein synthesis by hypoxia via activation of the endoplasmic reticulum kinase PERK and phosphorylation of the translation initiation factor eIF2alpha. Mol Cell Biol. 2002;22(21):7405-7416.

69. Bernardi RJ, Trump DL, Yu W-D, McGuire TF, Hershberger PA, Johnson CS. Combination of 1alpha,25-dihydroxyvitamin $\mathrm{D}$ (3) with dexamethasone enhances cell cycle arrest and apoptosis: role of nuclear receptor cross-talk and Erk/Akt signaling. Clin Cancer Res. 2001;7(12):4164-4173.

70. Kawauchi D, et al. A mouse model of the most aggressive subgroup of human medulloblastoma. Cancer Cell. 2012;21(2):168-180. 